\title{
LIFTING-LINE THEORY FOR A SWEPT WING AT TRANSONIC SPEEDS*
}

\author{
BY \\ L. PAMELA COOK \\ University of California, Los Angeles
}

Abstract. The boundary-value problems describing the first-order corrections

$$
O\left(\frac{1}{A R}, \frac{1}{A R} \ln A R\right)
$$

to two-dimensional flow about a lifting swept wing at transonic speeds $\left(M_{\infty}<1\right)$ are derived. The corrections are found by the use of the method of matched asymptotic expansions on the transonic small disturbance equations. The wing is at a sweep angle of $O\left(\left(1-M_{\infty}^{2}\right)^{1 / 2}\right)$ in the physical plane, hence of $O(1)$ in the transonic small disturbance plane. As has been noted for subsonic flow, the finite sweep angle necessitates the introduction of terms $O\left((A R)^{-1} \ln A R\right)$. These terms arise naturally in the matching process. Of particular interest is the derivation of the near field of a skewed lifting-line which is found by Mellin transform techniques. Also of interest is the fact that the influence of the nonzero sweep angle can be completely separated from the unswept solution.

Introduction. Prandtl's classical lifting-line theory gave aspect ratio corrections to the two-dimensional flow in the cross-sections of an unswept finite span wing. The induced downwash at each spanwise station was obtained. The method involved solving an integral equation. Van Dyke [13] systematized Prandtl's approach by recognizing that it could be considered as a singular perturbation. He considered the inviscid incompressible flow about an unswept wing with (aspect ratio) ${ }^{-1}$ as the small parameter. Inner and outer expansions were constructed and matched. The inner problem consists of two-dimensional flow at each spanwise station and corrections; the outer flow represents the flow past a bound vortex which sheds a vortex sheet and corrections. The advantage of this method is that there are no integral equations to be solved. Cook and Cole [6] extended Van Dyke's approach to describe compressible (transonic) flow about an unswept lifting wing. In this case the inner region represents the two-dimensional (nonlinear) transonic flow on a crosssection of the wing and corrections. The outer region represents the (linear) flow past a bound line vortex trailing a vortex sheet and corrections. It was shown that for similar airfoil sections the computations need be carried out at one spanwise station only since the corrections can be scaled to be independent of $z^{*}$.

In this paper we extend the techniques of Van Dyke, and Cook and Cole, to describe the transonic flow about a lifting, high aspect ratio, yawed wing. Investigations have been carried out on the swept wing for incompressible (linear) flow, for example Burgers [7],

* Received June 15, 1978. 
Thurber [12]. Thurber points out that a new term which is order of $(A R)^{-1} \ln (A R)$ arises in the induced downwash. A similar term arises naturally by the matching process in the transonic (compressible) flow. Cheng and $\mathrm{Hafez}[3]$ have treated swept wings in transonic flow. Their primary interest was in wings for which the (tangent of the sweep angle) ${ }^{3}$ is $\gg$ $\delta$, where $\delta \ll 1$ is the ratio of the thickness of the airfoil to its chord. In this paper we are concerned with the case where the (sweep angle) $)^{3}=O(\delta)$.

In Sec. 2 the three-dimensional transonic small disturbance theory boundary value problem for a lifting yawed wing is formulated [4]. The sweep angle is $O\left(\left(1-M_{\infty}^{2}\right)^{1 / 2}\right)$ in the physical plane, hence $O(1)$ in the small disturbance plane. In Sec. 3 the asymptotic expansions as $B=(A R) \delta^{1 / 3} \rightarrow \infty$, where $\delta$ is the typical flow deflection, are constructed. Shock waves can appear in the inner flow which describes the flow in cross-section planes and corrections. The outer expansion starts with the potential of a skewed bound vortex. The inner limit of the outer expansion is found in Appendix A by Mellin transform techniques. Matchings of the inner and outer expansions are shown. The boundary-value problems describing the inner potentials $\varphi_{0}, \varphi_{1}, \varphi_{2}$ are discussed in Sec. 4. $\varphi_{0}$ is described by the usual two-dimensional transonic small disturbance equation (in terms of coordinates measured from the wing). $\varphi_{1}$, which is the $O\left(A R^{-1} \ln A R\right)$ term, arises naturally from the matching process, and is described by a linear equation whose coefficients depend on $\varphi_{0}$ (variational equation). $\varphi_{2}$ is described by the same equation as $\varphi_{1}$ except that the equation is forced by spanwise variations in $\varphi_{0} \cdot \varphi_{2}$ can be broken up into two pieces, $\varphi_{2}{ }^{(1)}$ which is independent of sweep angle, and $\varphi_{2}{ }^{(2)}$ which is the sweep correction. Airfoils of similar section are discussed in Sec. 5. In particular, for similar sections the $\varphi_{0}, \varphi_{1}, \varphi_{2}{ }^{(1)}$ problems can be scaled to be independent of spanwise variation. Thus they need only be evaluated at one spanwise station.

There are nonuniformities in the expansion as $M_{\infty} \rightarrow 1$, as well as near the wing tips.

2. Formulation of boundary value problem. In this section we formulate a boundary value problem for the first-order transonic disturbance potential:

$$
\phi(x, \tilde{y}, \tilde{z} ; K, A, B) .
$$

In this restricted problem the tangent flow boundary condition is applied in the plane of the wing and the trailing vortex sheet is assumed to lie wholly in the plane of the wing. The Kutta-Joukowsky condition, that the flow leave the trailing edge smoothly, can thus be applied unambiguously at the trailing edge of the projection of the planform onto the plane of the wing. The self-induced downward motion and the rolling up of the trailing vortex sheet is not considered, so that presumably there is a non-uniformity of the approximation in the flow near the vortex wake toward downstream infinity. It is not expected that this will cause any appreciable error in the life distribution over the wing. In fact in the transonic region corresponding to high subsonic speeds, which is considered here, propagation of disturbances in the upstream direction is relatively weak. The boundary value problem formulated here is thus the transonic equivalent of tinearized lifting surface theory. The lifting-line theory which is constructed is the approximation for large aspect ratio.

Let the units of length be chosen so that the wing chord at the midspan is one and the extent of the wing in the $z$ direction is $2 b \cos \beta$ (see Fig. 2.1). The wing surface is then given by

$$
W(x, y, z)=0=y-\delta F_{u, l}(x-z \tan \Lambda, z / b)+\alpha x,
$$




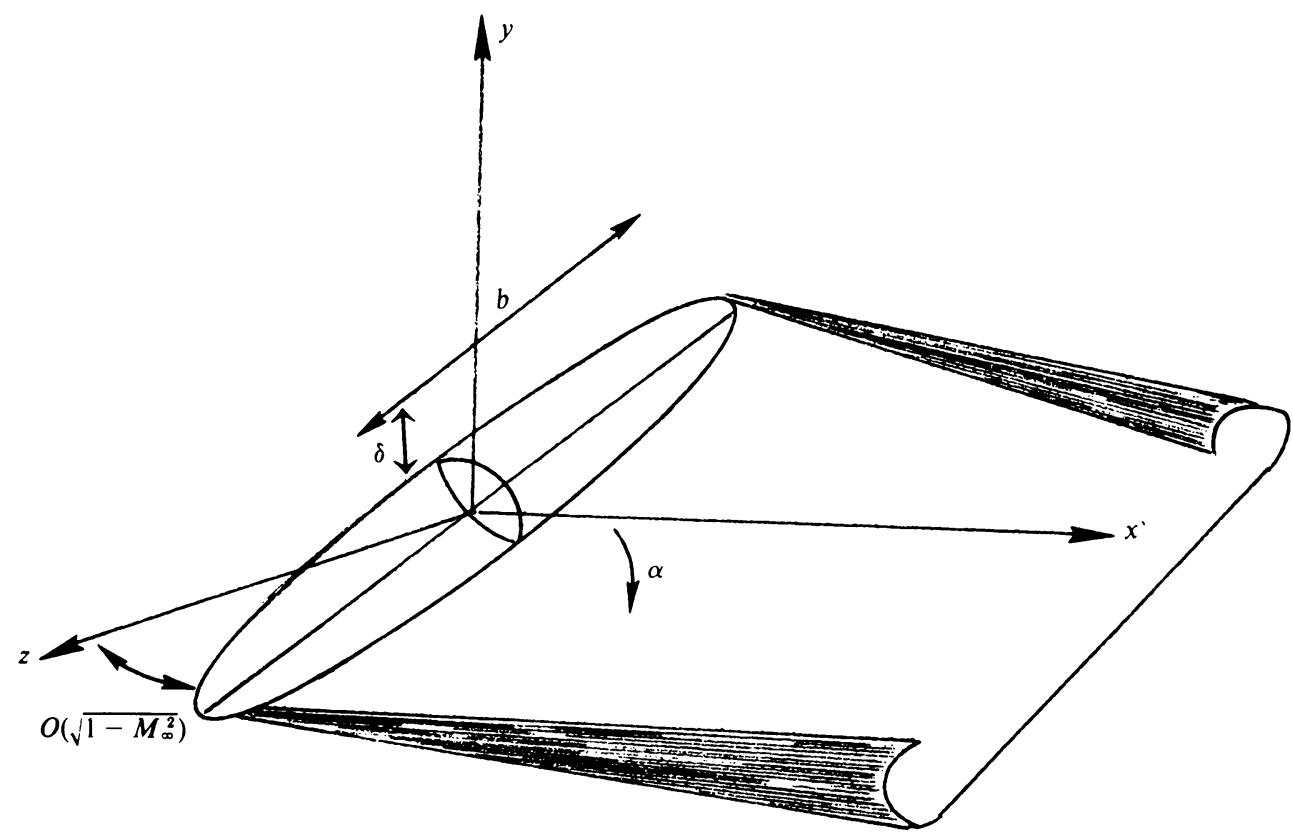

FIG. 2.1. Wing in physical coordinates.

where $\Lambda$ is such that the line $x=z \tan \Lambda$ joins the $z$ extremities of the wing, $F_{u, l}$ describes the geometry of the upper and lower surfaces respectively, $\alpha$ is the angle of attack, and $\delta$ the thickness ratio. The projection of the leading and trailing edges onto the plane $y=0$ is given by the functions $x_{L E, T E}(z / b)$.

The transonic expansion procedure is based on the limit process $\delta \downarrow 0, M_{\infty} \rightarrow 1$ (cf. [4]) with the transonic similarity parameter $K$, the angle of attack parameter $A$, the aspect ratio parameter $B$ all fixed. In addition, the coordinates $(x, \tilde{y}, \tilde{z})$ are held fixed, to account for the relatively large lateral extent of the disturbance field. Thus, the potential is represented as

$$
\begin{aligned}
\Phi(x, y, z ; M, \alpha, \delta, b) & =U\left\{x+\delta^{2 / 3} \phi(x, \tilde{y}, \tilde{z} ; K, A, B)\right. \\
& +\delta^{4 / 3} \phi^{(2)}(x, \tilde{y}, \tilde{z} ; K, A, B)+\cdots,
\end{aligned}
$$

where $K=$ transonic similarity parameter ${ }^{1}=\left(1-M_{\infty}^{2}\right) / \delta^{2 / 3}, A=$ angle of attack parameter $=\alpha / \delta, B=$ aspect ratio parameter $=b \delta^{1 / 3},\left(\tilde{y}=\delta^{1 / 3} y, \tilde{z}=\delta^{1 / 3} z\right)=$ transverse coordinates, and $\tan \beta=$ sweep angle $=\delta^{-1 / 3} \tan \Lambda$. (See Fig. 2.2).

Since shock waves introduce entropy changes only to the third order, it is sufficient to consider a potential $\Phi$. The first-approximation potential $\phi$ satisfies the well-known transonic equation

$$
\left(K-(\gamma+1) \phi_{x}\right) \phi_{x x}+\phi_{\tilde{y} \tilde{y}}+\phi_{\tilde{z} \tilde{z}}=0
$$

\footnotetext{
${ }^{1}$ In a more complete theory of the second transonic approximation, representations such as $M_{\infty}{ }^{2}=1-K \delta^{2 / 3}$ $+K_{2}(K) \delta^{4 / 3}-\cdots$ are used to describe the similarity parameter $K$. Similar representations are used for $(\tilde{y}, \tilde{z})$.
} 


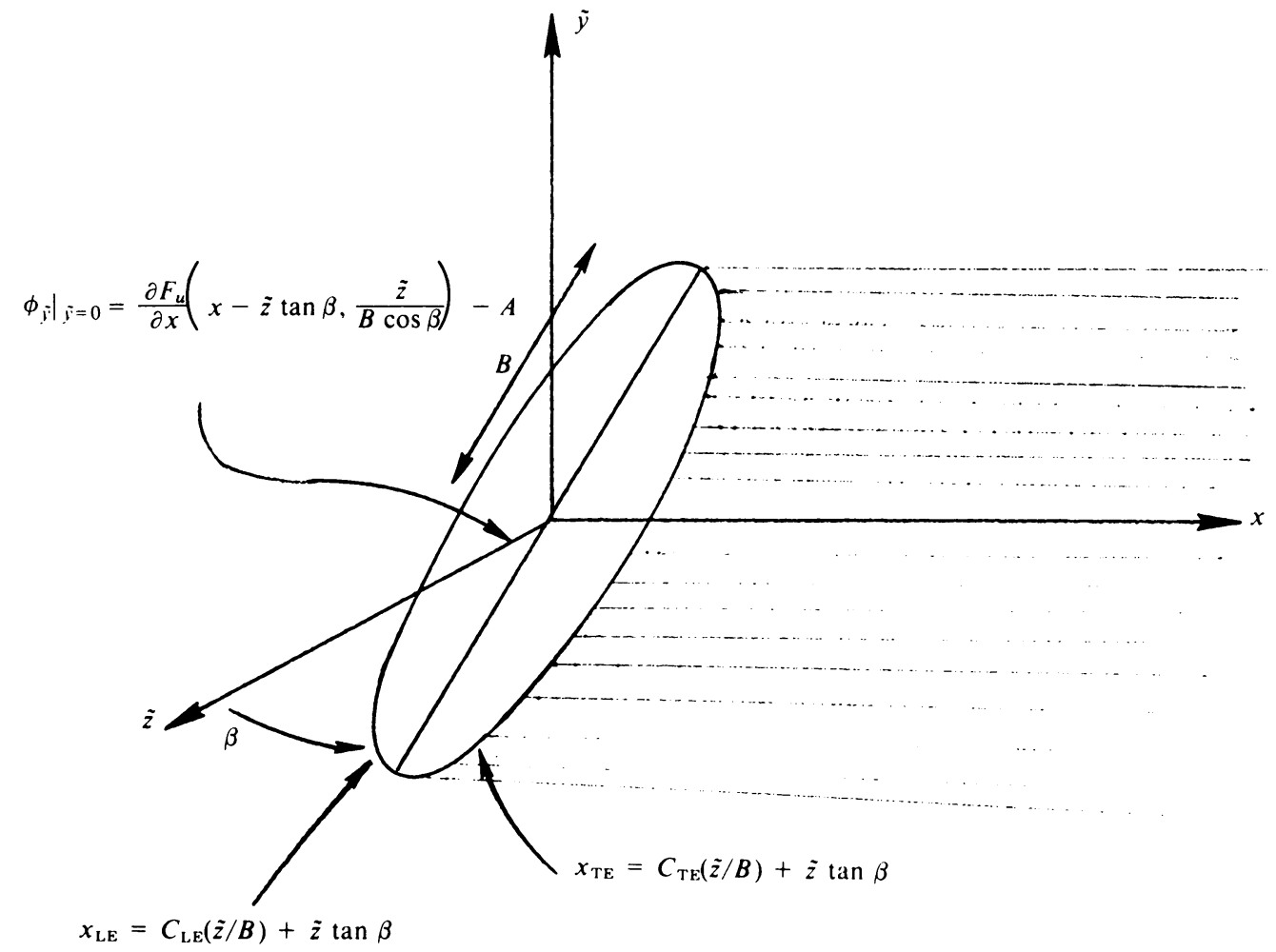

FIG. 2.2. Wing in transonic coordinates.

The boundary conditions for our problem will now be discussed. First of all there is no disturbance at upstream infinity, so that

$$
\phi_{x}, \phi_{\tilde{y}}, \phi_{z} \rightarrow 0 \text { as } x \rightarrow-\infty .
$$

Also, at downstream infinity pressure disturbances must die out. Since the transonic pressure coefficient is given by

$$
C_{p}=\frac{C_{p}}{\delta^{2 / 3}} \equiv \frac{p-p_{\infty}}{\left(\rho_{\infty} U^{2} / 2\right) \delta^{2 / 3}}=-2 \phi_{x},
$$

this means that

$$
\phi_{x} \rightarrow 0 \text { as } x \rightarrow \infty .
$$

The boundary condition of tangent flow becomes

$$
\phi_{\tilde{y}}(x, 0 \pm, \tilde{z})=\frac{\partial F_{u, l}}{\partial x}(x-\tilde{z} \tan \beta, \tilde{z} / B)-A,
$$

over $C_{L E}(\tilde{z} / B)<x-\tilde{z} \tan \beta<C_{T E}(\tilde{z} / B)$, where $\beta=\tan ^{-1}\left(\delta^{1 / 3} \tan \Lambda\right)$. At the trailing edge the Kutta-Joukowsky condition can be expressed

$$
\left[\phi_{x}\right]_{T E}=\phi_{x}\left(x_{T E}(\tilde{z} / B), 0+, \tilde{z}\right)-\phi_{x}\left(x_{T E}(\tilde{z}, B), 0-, \tilde{z}\right)=0 .
$$

Note that the Kutta-Joukowsky condition implies zero pressure perturbation at the trailing edge. 
The vortex sheet lies in the plane $\tilde{y}=0$, with $x-\tilde{z} \tan \beta>C_{T E}(\tilde{z} / B),|\tilde{z}|<B \cos \beta$, and stretches to downstream infinity. There is no jump in pressure across the vortex sheet, so

$$
\left[\phi_{x}\right]_{v s} \equiv \phi_{x}(x, 0+, \tilde{z})-\phi_{x}(x, 0-, \tilde{z})=0,
$$

or

$$
[\phi]_{{ }_{\theta}}=[\phi]_{T E}=\Gamma(\tilde{z}) \text { for }|\tilde{z}|<B \cos \beta .
$$

Here $\Gamma(\tilde{z})$ is the spanwise distribution of the (perturbation) circulation around a crosssection of the wing

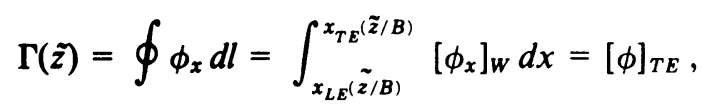

where $[\phi]_{W}=\phi_{x}(x, 0+, z)-\phi_{x}(x, 0-, z)$ for $|\tilde{z}|<B \cos \beta, C_{L E}<x-\tilde{z} \tan \beta<C_{T E}$. Note that the total lift $L$ of the wing is obtained by spanwise integration of the circulation distribution,

$$
L=\rho_{\infty} U^{2} \delta^{1 / 8} \iint_{W}\left[\phi_{x}\right] d x d \tilde{z}=\rho_{\infty} U^{2} \delta^{1 / 3} \int_{-B \cos \beta}^{B \cos \beta} \Gamma(\tilde{z}) d \tilde{z} .
$$

The problem for the potential $\phi$ is made complete by appending to the differential equation (2.3) the shock jump conditions which must hold across any shock waves which occur. Since (2.3) is a perturbation version of the continuity equation (the mass flux vector can be shown to have the expansion $\rho q=\rho_{\infty} U\left\{\mathrm{i}\left(1+\delta^{4 / 3}\left(K \phi_{x}-(\gamma+1) \phi_{x}^{2} / 2\right)+\cdots\right)+\right.$ $\left.\left.\delta \mathbf{q}_{\boldsymbol{T}}\right\}+\cdots\right)$ it can be written as a divergence,

$$
\frac{\partial}{\partial x}\left\{K \phi_{x}-\frac{\gamma+1}{2} \phi_{x}^{2}\right\}+\tilde{\nabla} \cdot \mathbf{q}_{T}=0,
$$

where $\tilde{\nabla}=(\partial / \partial \tilde{y}, \partial / \partial \tilde{z})=$ gradient in a transverse plane, $\mathbf{q}_{T}=\left(\phi_{\tilde{y}}, \phi_{\tilde{z}}\right)=$ velocity perturbation in a transverse plane. The integrated form of this divergence expression must hold across shock waves. If the shock surface is given by

$$
S(x, \tilde{y}, \tilde{z})=x-g(\tilde{y}, \tilde{z})=0,
$$

the local normal to the shock surface $\mathrm{n}$ is given by

$$
\mathrm{n} \cdot|\nabla \boldsymbol{S}|=\mathrm{i}-\delta^{1 / 3} \tilde{\nabla} \boldsymbol{g} \quad \text { in } \quad(x, \tilde{y}, \tilde{z}) \text { variables. }
$$

The integrated form of $(2.13)$ is

$$
\left[K \phi_{x}-\frac{\gamma+1}{2} \phi_{x}^{2}\right]_{s}-\left[\mathbf{q}_{T}\right] \cdot \tilde{\nabla} g=0,
$$

where []$_{s}=(($ downstream value $)-($ upstream value $))$ across the shock. Since there is no jump in tangential component of velocity across the shock it follows that $\left[\mathbf{q}_{T}\right]_{s} \times \tilde{\nabla} g=0$, or $\left[\mathbf{q}_{T}\right]$ is in the direction of $\tilde{\nabla} \mathbf{g}$. Also

$$
\left[\phi_{x}\right]_{s} \tilde{\nabla} g+\left[\mathbf{q}_{T}\right]_{s}=0,
$$

or alternatively

$$
[\phi]_{s}=0,
$$


guarantees no jump in tangential velocity component. (2.17) provides a differential expression for the shock geometry and a shock polar can be found from (2.16),

$$
\left[K \phi_{x}-\frac{\gamma+1}{2} \phi_{x}^{2}\right]_{s}\left[\phi_{x}\right]_{s}+[\tilde{\nabla} \phi]_{s}^{2}=0 \quad \text { (shock polar). }
$$

The differential equations, boundary conditions, and shock relations define a problem with a (presumably) unique solution. In order to obtain lifting-line theory we study the dependence of the solution of the problem on the aspect ratio parameter $\mathrm{B}$, which becomes large.

The formulation of the restricted lifting surface problem and the treatment of the lifting line as an approximation for $B \rightarrow \infty$ follow closely the ideas of Van Dyke for the analogous problem in incompressible flow.

3. Lifting-line expansions. In order to study the dependence of our first-order transonic disturbance potential $\phi(x, \tilde{y}, \tilde{z} ; K, A, B)$ on the aspect ratio $B \gg 1$, we consider the distinguished limit of the equation (2.3) under the boundary conditions (2.4), (2.6), (2.7), $(2.8)$, as $B \rightarrow \infty$. There are two basic limiting processes. In the outer limit $x^{*}=x / B, y^{*}=$ $\tilde{y} / B, z^{*}=\tilde{z} / B$ are held fixed as $B \rightarrow \infty$, and the planform shrinks to a line (of singularities). In the inner limit $\sigma=x-\tilde{z} \tan \beta, \tilde{y}, z^{*}=\tilde{z} / B$ are held fixed as $B \rightarrow \infty$, and so the boundary value problem for $\phi$ becomes essentially two-dimensional.

In the following section we summarize the form of the inner and outer expansions. Correctness of the expansions is shown by matching them, and the far fields of the inner expansion problems are found by the matching. Fig. 3.1 shows the wing geometry in the $\sigma$, $\tilde{y}, \tilde{z}$ coordinates.

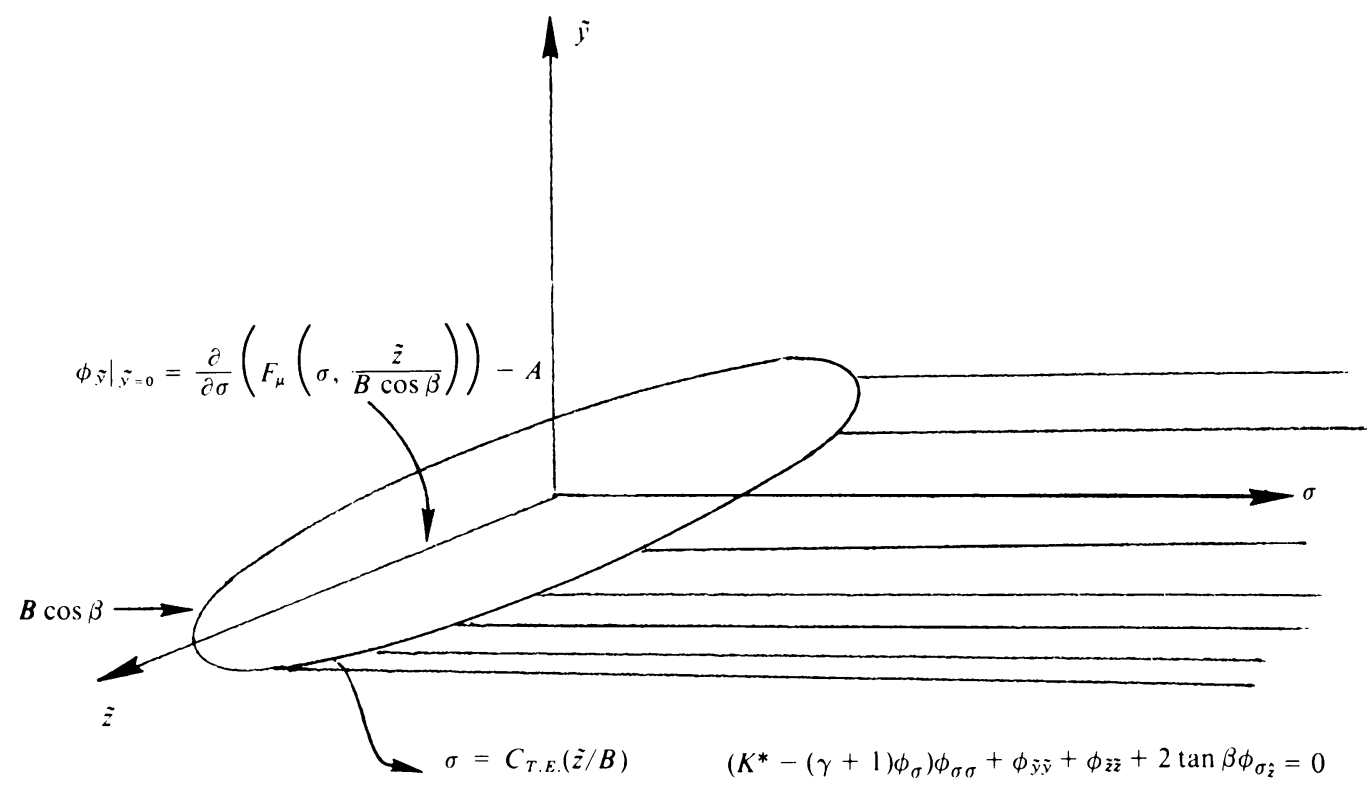

FIG. 3.1. Boundary value problem in $\sigma, \tilde{y}, \tilde{z}$ coordinates 
Inner expansion $\left(\sigma=x-\tilde{z} \tan \beta, \tilde{y}, z^{*}=\tilde{z} / B\right.$ fixed). The inner expansion has the form

$$
\phi(x, \tilde{y}, \tilde{z} ; B)=\phi_{d}\left(\sigma, \tilde{y} ; z^{*}\right)+\frac{\log B}{B} \phi_{1}\left(\sigma, \tilde{y} ; z^{*}\right)+\frac{1}{B} \phi_{2}\left(\sigma, \tilde{y} ; z^{*}\right)+\cdots
$$

where the equations governing the $\phi_{l}$ are

$$
\begin{gathered}
\left(K^{*}-(\gamma+1) \phi_{0 \sigma}\right) \phi_{0 \sigma \sigma}+\phi_{0 \tilde{y} \tilde{y}}=0, \\
\left(K^{*}-(\gamma+1) \phi_{0 \sigma}\right) \phi_{1 \sigma \sigma}-(\gamma+1) \phi_{0 \sigma \sigma} \phi_{1 \sigma}+\phi_{1 \tilde{y} \tilde{y}}=0, \\
\left(K^{*}-(\gamma+1) \phi_{0 \sigma}\right) \phi_{2 \sigma \sigma}-(\gamma+1) \phi_{0 \sigma \sigma} \phi_{2 \sigma}+\phi_{2 \tilde{y} \tilde{y}}=2 \tan \beta \phi_{0 \sigma z^{*}},
\end{gathered}
$$

where $K^{*}=K+\tan ^{2} \beta$. The tangent flow boundary conditions from (2.7) are:

$$
\begin{gathered}
\phi_{0 \hat{y}}\left(\sigma, 0 \pm ; z^{*}\right)=\frac{\partial F_{u, l}}{\partial \sigma}\left(\sigma, z^{*}\right)-A, \\
\phi_{1 y y}\left(\sigma, 0 \pm ; z^{*}\right)=0, \quad \phi_{2 y}\left(\sigma, 0 \pm ; z^{*}\right)=0,
\end{gathered}
$$

on $-\cos \beta<z^{*}<\cos \beta, C_{L E}\left(z^{*}\right)<\sigma<C_{T E}\left(z^{*}\right)$. The Kutta-Joukowsky condition must be satisfied for each term of the inner expansion:

$$
\left[\phi_{0 \sigma}\right]_{T E}=0, \quad\left[\phi_{1 \sigma}\right]_{T E}=0, \quad\left[\phi_{2 \sigma}\right]_{T E}=0,
$$

where the trailing edge is given by $-\cos \beta<z^{*}<\cos \beta, \sigma=C_{T E}\left(z^{*}\right)$. The conditions at infinity are obtained by matching to the near field of the outer expansion.

Note that the problem for $\phi_{0}$ has the form of the usual two-dimensional transonic problem for the flow past a lifting airfoil. At this point the influence of sweep angle is not felt. The correction term $\phi_{1}$, which is $O(\log B / B)$, enters solely because of a nonzero sweep angle and is needed for matching with the outer solution. Corrections for the finite aspect ratio appear in both $\phi_{1}$ and $\phi_{2}$ although more strongly in $\phi_{2}$ since some of the $\phi_{2}$ correction remains even in the case that the sweep angle goes to zero.

Outer expansion $\left(\sigma^{*}=\sigma / B=(x-\tilde{z} \tan \beta) / B, y^{*}=\tilde{y} / B, z^{*}=\tilde{z} / B=z / b\right.$ fixed as $B \rightarrow \infty)$. In this limit all lengths are measured relative to the span. The expansion has the form

$$
\phi(x, \tilde{y}, \tilde{z})=\varphi_{0}\left(x^{*}, y^{*}, z^{*}\right)+\frac{\log B}{B} \varphi_{1}\left(x^{*}, y^{*}, z^{*}\right)+\frac{1}{B} \varphi_{2}\left(x^{*}, y^{*}, z^{*}\right)+\cdots
$$

where the equations satisfied by the $\varphi_{t}$ are:

$$
\begin{gathered}
K^{*} \varphi_{0,1 \sigma^{*} \sigma^{*}}+\varphi_{0,1 y^{*} y^{*}}+\varphi_{0,1 z^{*} z^{*}}-2 \tan \beta \varphi_{0,1 \sigma^{*} z^{*}}=0, \\
K^{*} \varphi_{2 \sigma^{*} \sigma^{*}}+\varphi_{2 y^{*} y^{*}}+\varphi_{2 z^{*} z^{*}}-2 \tan \beta \varphi_{2 \sigma^{*} z^{*}}=(\gamma+1) \varphi_{0 \sigma^{*}} \varphi_{0 \sigma^{*} \sigma^{*}} .
\end{gathered}
$$

As in the inner expansion the $O(\log B / B)$ term is introduced for matching. However, in the outer expansion the $O(\log B / B)$ terms remain even in the case that the sweep angle goes to zero. Note that, if written in $x^{*}, y^{*}, z^{*}$ variables, Eqs. $(3.5,3.5 \mathrm{a}, 3.5 \mathrm{~b})$ are precisely the same as for the unswept wing. The nonlinearity appears in the $O(1 / B)$ term and the dependence of the outer expansion on sweep angle appears from boundary conditions and matching. For a fixed $\sigma, \sigma^{*} \rightarrow 0$ as $B \rightarrow \infty$, hence the image of the wing collapses to a line. Its effect on the outer flow can be represented in the $x^{*}, y^{*}, z^{*}$ coordinates as a skewed line vortex shedding its vorticity downstream and higher order singularities. The boundary conditions on the $\varphi_{l}$ are 


$$
\begin{aligned}
& \varphi_{1 \sigma^{*}} \underset{r^{* \rightarrow \infty}}{\longrightarrow} 0, \\
& {\left[\varphi_{i \sigma^{\prime}}\right]_{v s}=0 .}
\end{aligned}
$$

The remaining conditions are obtained by matching with the far field of the inner expansion. This matching determines the type of singularities needed in the $\varphi_{i}$. We find that:

$$
\begin{aligned}
\varphi_{0}= & \frac{1}{4 \pi} \int_{-1}^{1} \frac{y^{*} \gamma(s)}{y^{* 2}+\left(z^{*}-s \cos \beta\right)^{2}}\left(1+\frac{x^{*}-s \sin \beta}{\sqrt{\left(x^{*}-s \sin \beta\right)^{2}+K y^{* 2}+K\left(z^{*}-s \cos \beta\right)^{2}}}\right) d s \\
= & \frac{1}{4 \pi} \int_{-1}^{1} \frac{y^{*} \gamma(s)}{y^{* 2}+\left(z^{*}-s \cos \beta\right)^{2}} \\
& \left(1+\frac{\sigma^{*}+\left(z^{*}-s \cos \beta\right) \tan \beta}{\sqrt{K^{*}\left(z^{*}-s \cos \beta\right)^{2}+2 \sigma^{*}\left(z^{*}-s \cos \beta\right) \tan \beta+K_{y^{* 2}+\sigma^{* 2}}}}\right) d s
\end{aligned}
$$

which is the superposition of elementary horseshoe vortices distributed along $x^{*}=z^{*}$ $\tan \beta,-1<z^{*} / \cos \beta<1$, and trailing off parallel to the $\sigma^{*}$ axis,

$$
\varphi_{1}=\frac{\sigma^{*}}{4 \pi} \frac{K}{K^{*}} \int_{-1}^{1} \frac{D_{1}(s)}{\left[K^{*}\left(z^{*}-s \cos \beta\right)^{2}+2 \sigma^{*}\left(z^{*}-s \cos \beta\right) \tan \beta+K y^{* 2}+\sigma^{* 2}\right]^{3 / 2}} d s
$$

which is the potential in $\sigma^{*}, y^{*}, z^{*}$ coordinates of a distribution of divortices along the lifting line, and

$$
\begin{aligned}
\varphi_{2}= & \frac{\sigma^{*}}{4 \pi} \frac{K}{K^{*}} \int_{-1}^{1} \frac{D_{2}(s)}{\left[K^{*}\left(z^{*}-s \cos \beta\right)^{2}+2 \sigma^{*}\left(z^{*}-s \cos \beta\right) \tan \beta+K y^{* 2}+\sigma^{* 2}\right]^{3 / 2}} d s \\
& +\frac{y^{*}}{4 \pi} \frac{K}{K^{*}} \int_{-1}^{1} \frac{\varepsilon_{2}(s)}{\left[K^{*}\left(z^{*}-s \cos \beta\right)^{2}+2 \sigma^{*}\left(z^{*}-s \cos \beta\right) \tan \beta+K y^{* 2}+\sigma^{* 2}\right]^{3 / 2}} d s \\
& +\frac{y^{*}}{4 \pi} \int_{-1}^{1} \frac{\gamma_{2}(s)}{y^{*}+\left(z^{*}-s \cos \beta\right)^{2}} \\
& \cdot\left(1+\frac{\sigma^{*}+\left(z^{*}-s \cos \beta\right) \tan \beta}{\sqrt{K^{*}\left(z^{*}-s \cos \beta\right)^{2}+2 \sigma^{*}\left(z^{*}-s \cos \beta\right) \tan \beta+K y^{* 2}+\sigma^{* 2}}}\right) d s+\varphi_{2}^{P}
\end{aligned}
$$

where $\varphi_{2}^{P}$ is a particular solution of $(3.5 \mathrm{~b})$ whose behavior is specified as $\left(\sigma^{*}, y^{*}\right) \rightarrow 0$, $\sigma^{*} / y^{*}$ fixed.

Expansions of the integrals in $(3.8,3.9,3.10)$ are worked out in general in Appendix A. In particular it is found that

$$
\begin{aligned}
\varphi_{0}= & -\left(\frac{\gamma_{d}\left(z^{*} / \cos \beta\right)}{2 \pi \cos \beta}\right) \theta+\frac{\tan \beta}{\sqrt{ } K^{*}}\left(\frac{\gamma_{0}^{\prime}}{2 \pi \cos \beta}\right) y^{*} \ln r^{*}-J_{0}\left(z^{*}\right) y^{*}+O\left(y^{* 2} \ln r^{*}\right), \\
\varphi_{1}= & \frac{\sigma^{*}}{2 \pi \sqrt{ } K^{*} r^{* 2}} \frac{D_{1}\left(z^{*} / \cos \beta\right)}{\cos \beta}+\frac{\gamma_{1}}{2 \pi} \theta+\tan \beta \frac{D_{1}^{\prime}(\cos 2 \theta+1)}{4 \pi\left(K^{*}\right)^{3 / 2} \cos \beta}+O\left(y^{*} \ln r^{*}\right), \\
\varphi_{2}= & \frac{\sigma^{*}}{2 \pi \sqrt{ } K^{*} r^{* 2}} \frac{D_{2}\left(z^{*} / \cos \beta\right)}{\cos \beta}+\frac{y^{*}}{2 \pi \sqrt{ } K^{*} r^{* 2}} \frac{\varepsilon_{2}\left(z^{*} / \cos \beta\right)}{\cos \beta} \\
& +\tan \beta\left\{\frac{D_{2}^{\prime}(\cos 2 \theta+1)}{4 \pi\left(K^{*}\right)^{3 / 2}}+\frac{\varepsilon_{2}^{\prime} \sin 2 \theta}{4 \pi\left(K^{*}\right)^{3 / 2}}\right\}-\frac{\gamma_{2}}{2 \pi \cos \beta} \theta+\varphi_{2}^{P}+O\left(y^{*} \ln y^{*}\right),
\end{aligned}
$$


where

$$
J_{0}=+\frac{1}{4 \pi \cos \beta} \int_{-\cos \beta}^{\cos \beta} \frac{\gamma_{0}^{\prime}(s / \cos \beta)}{z^{*}-s} d s+J_{0}^{c}\left(z^{*}\right),
$$

$J_{0}^{c}\left(z^{*}\right)$ is given in Appendix $A$ (A.9), ( $)^{\prime}$ means $\partial / \partial z^{*}(), \theta=\arctan \left(\sqrt{ } K^{*} y^{*} / \sigma^{*}\right)$, $r^{*}=\left(K^{*} y^{* 2}+\sigma^{* 2}\right)^{1 / 2}$ and

$$
\begin{aligned}
& \varphi_{2}^{P}=\frac{\gamma+1}{4 K^{*}}\left(\frac{\gamma_{0}}{2 \pi \cos \beta}\right)^{2}\left\{\frac{\log r^{*}}{r^{*}} \cos \theta-\frac{\cos 3 \theta}{4 r^{*}}\right\} \\
& +\tan \beta \frac{\gamma+1}{4 K^{*}}\left(\left(\frac{\gamma_{0}}{2 \pi \cos \beta}\right)^{2}\right)^{\prime}\left\{\frac{+\cos (2 \theta+1)}{2} \log r^{*}+\frac{\theta^{2}}{2}+\frac{\cos 2 \theta}{8}-\frac{1}{8} \cos 4 \theta\right\} .
\end{aligned}
$$

Matching. The boundary value problem described by the nonlinear equation (3.2a) and its associated boundary conditions $(3.3 \mathrm{a}, 3.4 \mathrm{a})$ has the form of the boundary value problem for two-dimensional transonic flow past a lifting airfoil. In particular the far field is given by [5]

$$
\begin{aligned}
\phi_{0}= & -\frac{\Gamma_{0} \theta}{2 \pi}+\frac{(\gamma+1)}{4 K^{*}}\left(\frac{\Gamma_{0}}{2 \pi}\right)^{2} \frac{\log r}{r} \cos \theta+\frac{1}{r}\left\{\frac{D_{0}}{2 \pi \sqrt{ } K^{*}} \cos \theta+\frac{E_{0}}{2 \pi \sqrt{ } K^{*}} \sin \theta\right. \\
& \left.-\frac{1}{16} \frac{(\gamma+1)}{K^{*}}\left(\frac{\Gamma_{0}}{2 \pi}\right)^{2} \cos 3 \theta\right\}+O\left(\frac{\log r}{r^{2}}\right) \text { as } r \rightarrow \infty,
\end{aligned}
$$

where $r=\left(\sigma^{2}+K^{*} y^{* 2}\right)^{1 / 2}, \theta=\tan ^{-1}\left(\sqrt{ } K^{*} \tilde{y} / \sigma\right)$, and $\Gamma_{d}\left(z^{*}\right)$ is the circulation at the spanwise station $z^{*} ; D_{0}\left(z^{*}\right)$ and $E_{0}\left(z^{*}\right)$ are the doublet strengths at the station $z^{*}$.

The behavior of $\phi_{1}, \phi_{2}$ as $r \rightarrow \infty$ is one of the main results of matching. In particular we expect that

$$
\phi_{1}=A_{1}\left(z^{*}\right) \sigma+B_{1}\left(z^{*}\right) \tilde{y}+\frac{\Gamma_{0}}{2 \pi} \frac{A_{1}}{4} \sin 2 \theta-\frac{\Gamma_{1}}{2 \pi} \theta+\cdots ;
$$

that is, a uniform flow, then a circulation term. The term with $\sin 2 \theta$ arises naturally in Eq. (3.2b). We also find that

$$
\begin{aligned}
\phi_{2}= & \frac{\tan \beta}{\sqrt{ } K^{*}} \frac{\Gamma_{0}^{\prime}}{2 \pi} \tilde{y} \log r+B_{2} \tilde{y}+A_{2} \sigma-\frac{\Gamma_{2} \theta}{2 \pi}+\frac{\gamma+1}{4} A_{2} \frac{\Gamma_{0}^{\prime}}{2 \pi} \sin 2 \theta \\
& +\tan \beta\left\{\left(\frac{\gamma+1}{4 K^{*}}\left(\left(\frac{\Gamma_{0}}{2 \pi}\right)^{2}\right)^{\prime}\right)\left(\frac{\log r(\cos 2 \theta+1)}{2}+\frac{\theta^{2}}{2}+\frac{\cos 2 \theta}{8}-\frac{\cos 4 \theta}{8}\right)\right. \\
& \left.+\frac{D_{0}^{\prime}}{2 \pi\left(K^{*}\right)^{3 / 2}} \frac{\cos 2 \theta+1}{2}+\frac{E_{0}^{\prime}}{2 \pi\left(K^{*}\right)^{3 / 2}} \frac{\sin 2 \theta}{2}\right\}+O\left(\frac{1}{r} \log r\right),
\end{aligned}
$$

as $r \rightarrow \infty$.

The term $O(\tilde{y} \log r)$ arises from the forcing part of the equation, $\phi_{0 \sigma z^{*}}$. In the matching this term combines with the $B_{1} \tilde{y}$ term in $\phi_{1}$. The $A_{2}, B_{2}$ terms represent a possible uniform flow, $\Gamma_{2}$ a circulation, and the $O(1)$ terms multiplied by $\tan \beta$ arise from higher-order forcing terms in $\phi_{0 \sigma z^{*}}$ as well as from $\phi_{0 \sigma} \phi_{2 \sigma \sigma}$ and $\phi_{2 \sigma} \phi_{0 \sigma \sigma}$ terms.

Matching is carried out in each cross-section plane $z^{*}$ fixed, $\left|z^{*}\right|^{\sigma}<\cos \beta$, with the help of an intermediate limit. In a class of limits intermediate to the inner and outer limits,

$$
r_{\lambda}=\frac{r}{\lambda(B)}=\frac{\left(\sigma^{2}+K^{*} \tilde{y}^{2}\right)^{1 / 2}}{\lambda(B)} \text { is fixed as } B \rightarrow \infty,
$$


where $1 \ll \lambda \ll B$. Thus in the intermediate limit

$$
r=\lambda(B) r_{\lambda} \rightarrow \infty, \quad r^{*}=\frac{r}{B}=\frac{\lambda(B)}{B} r_{\lambda} \rightarrow 0 .
$$

The calculations are simplified if the far-field of the inner expansion is merely written in terms of outer ()$^{*}$ coordinates and a direct comparison of the inner $\left(r^{*} \rightarrow \infty\right)$ and outer expansion $\left(r^{*} \rightarrow 0\right)$ is made.

Now, writing $r=B r^{*}$ in (3.14), (3.15) and (3.16), the expansions take the form:

$$
\text { Inner: } \begin{aligned}
\phi= & -\frac{\Gamma_{0}}{2 \pi} \theta+\frac{\tan \beta}{\sqrt{ } K^{*}} \frac{\Gamma_{0}^{\prime}}{2 \pi} y^{*} \log r^{*}+B_{2} y^{*}+A_{2} \sigma^{*} \\
& +\frac{\log B}{B}\left\{\frac{\gamma+1}{4 K^{*}}\left(\frac{\Gamma_{0}}{2 \pi}\right)^{2} \frac{\cos \theta}{r^{*}}-\frac{\Gamma_{1} \theta}{2 \pi}+\tan \beta \frac{\gamma+1}{4 K^{*}}\left(\left(\frac{\Gamma_{0}}{2 \pi}\right)^{2}\right)^{\prime} \frac{\cos 2 \theta+1}{2}\right\} \\
& +\frac{1}{B}\left\{\frac{\gamma+1}{4 K^{*}}\left(\frac{\Gamma_{0}}{2 \pi}\right)^{2} \frac{\log r^{*}}{r^{*}} \cos \theta-\frac{\gamma+1}{4 K^{*}}\left(\frac{\Gamma_{0}}{2 \pi}\right)^{2} \frac{\cos 3 \theta}{4 r^{*}}\right. \\
& +\frac{D_{0}}{2 \pi \sqrt{ } K^{*}} \frac{\cos \theta}{r^{*}}+\frac{E_{0}}{2 \pi \sqrt{ } K^{*}} \frac{\sin \theta}{r^{*}}-\frac{\Gamma_{2} \theta}{2 \pi}+\frac{\gamma+1}{4} A_{2} \frac{\Gamma_{0}^{\prime}}{2 \pi} \sin 2 \theta \\
& +\tan \beta\left(\left(\frac{\gamma+1}{4 K^{*}}\right)\left(\left(\frac{\Gamma_{0}}{2 \pi}\right)^{2}\right)^{\prime}\left(\frac{\log r^{*}(\cos 2 \theta+1)}{2}+\frac{\theta^{2}}{2}+\frac{\cos 2 \theta}{8}-\frac{\cos 4 \theta}{8}\right)\right. \\
& \left.\left.+\frac{D_{0}^{\prime}}{2 \pi\left(K^{*}\right)^{3 / 2}} \frac{\cos 2 \theta+1}{2}+\frac{E_{0}^{\prime}}{2 \pi\left(K^{*}\right)^{3 / 2}} \frac{\sin 2 \theta}{2}\right)\right\}
\end{aligned}
$$

where we chose $B_{1}=\left(\tan \beta / \sqrt{ } K^{*}\right)\left(\Gamma_{0}^{\prime} / 2 \pi\right)$ in order for the expansion to remain valid. Also we chose $A_{1}=0$; the justification for that will be that the expansions match.

$$
\text { Outer: } \begin{aligned}
\phi= & -\left(\frac{\gamma_{0}}{2 \pi \cos \beta}\right) \theta+\tan \beta\left(\frac{\gamma_{0}^{\prime}}{2 \pi \cos \beta}\right) \frac{1}{\sqrt{ } K^{*}} y^{*} \ln r^{*}-J_{0} y^{*} \\
& +\frac{\log B}{B}\left\{\frac{D_{1}}{2 \pi \sqrt{ } K^{*} \cos \beta} \frac{\cos \theta}{r^{*}}-\left(\frac{\gamma_{1}}{2 \pi \cos \beta}\right) \theta+\frac{D_{1}^{\prime}}{4 \pi\left(K^{*}\right)^{3 / 2}} \frac{\tan \beta}{\cos \beta}(\cos 2 \theta+1)\right\} \\
& +\frac{1}{B}\left\{\left(\frac{\gamma+1}{4 K^{*}}\right)\left(\frac{\gamma_{0}}{2 \pi \cos \beta}\right)^{2} \frac{\log r^{*}}{r^{*}} \cos \theta-\frac{\gamma+1}{16 K^{*}}\left(\frac{\gamma_{0}}{2 \pi \cos \beta}\right)^{2} \frac{\cos 3 \theta}{r^{*}}\right. \\
& +\frac{D_{2}}{2 \pi \sqrt{ } K^{*} \cos \beta} \frac{\cos \theta}{r^{*}}+\frac{\varepsilon_{2}}{2 \pi \sqrt{ } K^{*} \cos \beta} \frac{\sin \theta}{r^{*}}-\frac{\gamma_{2}}{2 \pi \cos \beta} \theta \\
& +\tan \beta\left(\frac{\gamma+1}{4 K^{*}}\left(\left(\frac{\gamma_{0}}{2 \pi \cos \beta}\right)^{2}\right)^{\prime}\left(\frac{(\cos 2 \theta+1) \log r^{*}}{2}+\frac{\theta^{2}}{2}+\frac{\cos 2 \theta}{8}-\frac{\cos 4 \theta}{8}\right)\right. \\
& \left.\left.+\frac{D_{2}^{\prime}(\cos 2 \theta+1)}{4 \pi\left(K^{*}\right)^{3 / 2}}+\frac{\varepsilon_{2}^{\prime} \sin 2 \theta}{4 \pi\left(K^{*}\right)^{3 / 2}}\right)\right\}
\end{aligned}
$$

Matching is accomplished to all orders shown if

$$
\begin{gathered}
\frac{\gamma_{0}\left(z^{*} / \cos \beta\right)}{\cos \beta}=\Gamma_{0}\left(z^{*}\right), \frac{D_{1}}{\cos \beta}=\frac{\gamma+1}{4 \sqrt{ } K^{*}} \frac{\left(\Gamma_{0}\left(z^{*}\right)\right)^{2}}{2 \pi}, \frac{\mathcal{E}_{2}}{\cos \beta}=E_{0}, \\
B_{2}=-J_{0}\left(z^{*}\right), \frac{\gamma_{1}}{\cos \beta}=\Gamma_{1}\left(z^{*}\right), \frac{\gamma_{2}}{\cos \beta}=\Gamma_{2}\left(z^{*}\right), A_{2}=0, \frac{D_{2}}{\cos \beta}=D_{0} .
\end{gathered}
$$


The essential matching for the completion of the boundary value problems for $\phi_{1}, \phi_{2}$ defines $B_{1}\left(z^{*}\right)$ and $B_{2}\left(z^{*}\right)$ in terms of the first inner circulation $\Gamma_{0}\left(z^{*}\right)$. For $\beta=0, B_{1}=0$, and $B_{2}$ agrees with the induced downwash of the trailing vortex sheet as in the classical lifting-line theory.

4. Boundary value problems for $\phi_{0}, \phi_{1}, \phi_{2}$. As a result of the asymptotic matching of the previous section, complete boundary value problems can be formulated for the first-, second- and third-order inner potentials $\phi_{0}, \phi_{1}, \phi_{2}$. The problem for $\phi_{0}$ is the twodimensional flow past an airfoil at the same shape and angle of attack as the actual wing at a given $z^{*}$ station. The $O(\log B / B)$ correction, $\phi_{1}$, corresponds to a perturbed twodimensional flow past a flat plate with induced downwash at infinity, due to the trailing vortex sheet. The $O(1 / B)$ correction, $\phi_{2}$, is a new type of term. It corresponds to the flow past a flat plate with an induced $O(y \ln y)$-type behavior at infinity. There are also special shock conditions to be considered for each of $\phi_{1}, \phi_{2}$.

For $\phi_{0}$ we have (see (3.2a), (3.3a), (3.4a), (3.14))

$$
\left(\left(K^{*}-(\gamma+1)\right) \phi_{0 \sigma}\right) \phi_{0 \sigma \sigma}+\phi_{0 \tilde{y} y}=0,
$$

with the following boundary conditions:

$$
\begin{gathered}
\phi_{0 \sigma} \rightarrow \underset{\sigma}{0} \text { at infinity, } \\
\phi_{0 \tilde{y}}\left(\sigma, 0 \pm, z^{*}\right)=\frac{\partial F_{u, l}}{\partial \sigma}\left(\sigma, z^{*}\right)-A,
\end{gathered}
$$

the Kutta-Joukowsky condition

$$
\left[\phi_{\omega_{\sigma}}\right]_{\mathrm{TE}}=0,
$$

and the shock conditions which (cf. (B.8, B.9)) are integral forms of the conservation form of (4.1):

$$
\left[K \phi_{0 \sigma}-\frac{\gamma+1}{2} \phi_{0 \sigma}^{2}\right]+\left[\phi_{0 \tilde{y}}\right]^{2}=0, \quad\left[\phi_{0}\right]=0,
$$

on the first-order shock locus

$$
S_{0}: \sigma=g_{0}(\tilde{y})
$$

The shock geometry is such that

$$
g_{0}^{\prime}(\tilde{y})=-\left[\phi_{0 \tilde{y}}\right] /\left[\phi_{0 \sigma}\right] .
$$

These shock wave jump conditions must apply locally across any shock waves that appear in supersonic zones of the solution. The shock locus $g_{0}(\tilde{y})$ is not known in advance and must be found as part of the solution. Fig. 4.1 shows the boundary value problem for $\phi_{0}$.

For a given airfoil shape the boundary value problem for $\phi_{0}$ cannot, in general, be solved analytically. Instead, one of the standard computational schemes for resolving shock waves is used $([2,9])$. Alternatively one could consider shock-free transonic flows and the corresponding airfoils generated from the hodograph solutions, for example Garabedian [8].

The correction flow $\phi_{1}$ satisfies a boundary value problem which is linear, assuming $\phi_{0}, g_{0}$ are known. The equation is $(3.2 \mathrm{~b})$

$$
\left(K^{*}-(\gamma+1) \phi_{0 \sigma}\right) \phi_{1 \sigma \sigma}-(\gamma+1) \phi_{1 \sigma} \phi_{0 \sigma \sigma}+\phi_{1 \tilde{y} \tilde{y}}=0
$$




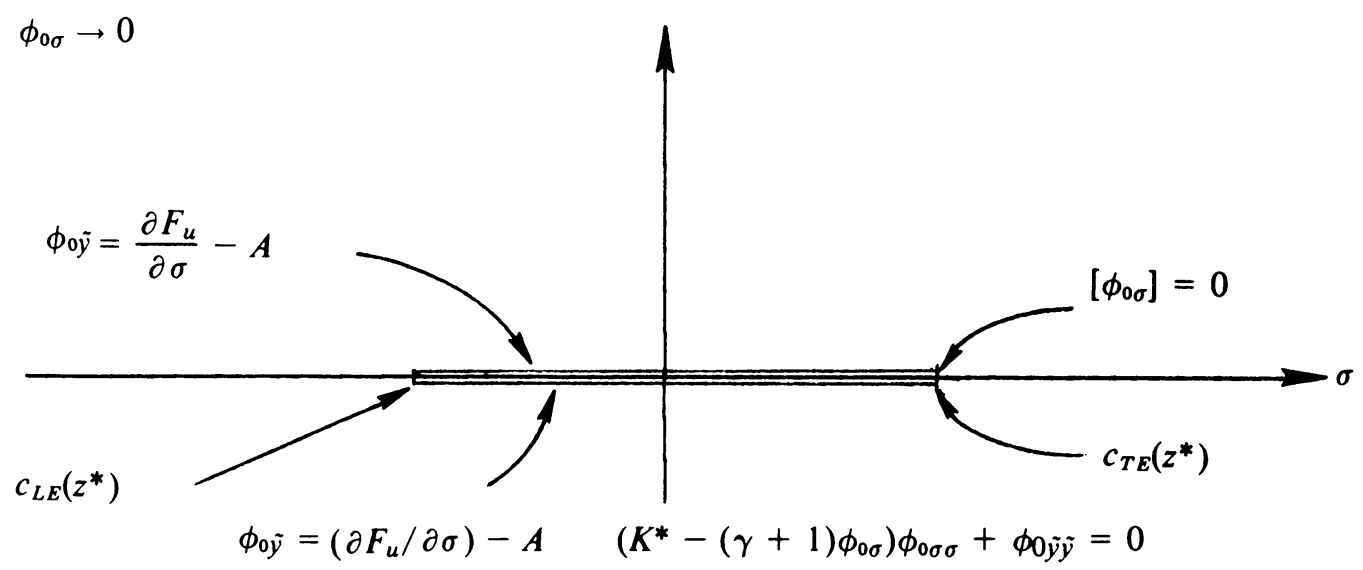

Fig. 4.1. Boundary value problem for $\phi_{0}$.

or in conservation form

$$
\left(K^{*} \phi_{1 \sigma}-(\gamma+1) \phi_{0 \sigma} \phi_{1 \sigma}\right)_{\sigma}+\phi_{1 \tilde{y} \tilde{y}}=0 .
$$

The boundary condition on the slit $\sigma^{*}=0, C_{\mathrm{LE}}\left(z^{*}\right)<\sigma<C_{\mathrm{TE}}\left(z^{*}\right)$ is that of a flat plate

$$
\phi_{1 \tilde{y}}\left(\sigma, 0 \pm, z^{*}\right)=0 .
$$

However, there is a downwash at infinity toward the flat plate, as determined by matching (3.17), (3.18). We have

$$
\phi_{1}=-\tilde{y}\left(\tan \beta \frac{\Gamma_{0}^{\prime}}{2 \pi \sqrt{ } K^{*}}\right)-\frac{\Gamma_{1}\left(z^{*}\right)}{2 \pi} \theta+\mathrm{O}\left(\frac{1}{r} \ln r\right)
$$

as $r \rightarrow \infty$, where $\Gamma_{1}\left(z^{*}\right)$, the induced circulation, is to be found. The boundary conditions on the trailing edge and wake are identical to those of $\phi_{0}$ :

$$
\begin{gathered}
{\left[\phi_{1_{\sigma}}\right]_{\mathrm{TE}}=0 \quad(\text { Kutta-Joukowsky condition }),} \\
{\left[\phi_{1}\right]_{\text {wake }}=\text { const. }=\Gamma_{1}\left(z^{*}\right) .}
\end{gathered}
$$

The shock conditions for the linear equation require special treatment. Details are given in Appendix B. The results are

$$
\begin{gathered}
{\left[K \phi_{0 \sigma}-\frac{\gamma+1}{2} \phi_{0 \sigma}^{2}\right]\left[\phi_{1 \sigma}\right]+\left[\phi_{0 \sigma}\right]\left[K^{*} \phi_{1 \sigma}-(\gamma+1) \phi_{0 \sigma} \phi_{1 \sigma}\right]+2\left[\phi_{0 \tilde{y}}\right]\left[\phi_{1 \tilde{y}}\right]} \\
=-g_{1}(\tilde{y})\left\{\left[\phi_{0 \sigma}\right]\left[\phi_{0 \sigma \sigma}-\frac{\gamma+1}{2} \phi_{0 \sigma}^{2}\right]+\left[\phi_{0 \sigma}\right]\left[K^{*} \phi_{0 \sigma \sigma}+(\gamma+1) \phi_{0 \sigma} \phi_{0 \sigma \sigma}\right]+2\left[\phi_{0 \sigma \tilde{y}}\right]\right\}, \\
{\left[\phi_{1}\right]=-g_{1}\left[\phi_{0 \sigma}\right],}
\end{gathered}
$$

where all jumps are applied on the zeroth-order locus $\sigma=g_{0}(\tilde{y})$. The shock locus has been represented as

$$
x=g_{0}(\tilde{y})+\frac{\log B}{B} g_{1}\left(\tilde{y} ; z^{*}\right)+\frac{1}{B} g_{2}\left(\tilde{y} ; z^{*}\right)+\cdots .
$$


An overall conservation theorem has also been derived to act as a supplementary shock condition:

$$
\int_{s_{0}}\left\{\left[w^{*} \phi_{1 \sigma}\right]-\frac{\left[\phi_{0 \tilde{y}}\right]}{\left[\phi_{0 x}\right]}\left[\phi_{1 \tilde{y}}\right]\right\} d \tilde{y}=0
$$

where $w^{*}=-K^{*}+(\gamma+1) \phi_{0 \sigma}$. This condition guarantees that there are no source terms at infinity in the solution to the boundary value problem for $\phi_{1}$. The equation to be solved (4.8) is a mixed-type equation, but it is linear and the regions of ellipticity or hyperbolicity are known in advance from the $\phi_{0}$ solution. Fig. 4.2 illustrates the boundary value problem for $\phi_{1}$.

The second correction to the two-dimensional flow, $\phi_{2}$, satisfies a linear equation which is forced by the zeroth-order flow. The equation for $\phi_{2}$ is

$$
\left(K^{*}-(\gamma+1) \phi_{0 \sigma}\right) \phi_{2 \sigma \sigma}-(\gamma+1) \phi_{0 \sigma \sigma} \phi_{2 \sigma}+\phi_{2 \tilde{y} \tilde{y}}=2 \tan \beta \phi_{0 \sigma z}{ }^{*},
$$

where $\phi_{0}$ is assumed known. The boundary condition on the slit $\tilde{y}=0, C_{\mathrm{LE}}\left(z^{*}\right)<\sigma<$ $C_{\mathrm{TE}}\left(z^{*}\right)$ is that of a flat plate,

$$
\phi_{2 \tilde{y}}\left(\sigma, 0 \pm ; z^{*}\right)=0 .
$$

The condition at infinity, which is determined by matching, is:

$$
\begin{aligned}
\phi_{2} \sim & \tan \beta \frac{\Gamma_{0}^{\prime}}{2 \pi K^{*}} \tilde{y} \log r-J_{0}\left(z^{*}\right) \tilde{y} \\
& +\tan \beta\left\{\left(\frac{\gamma+1}{4 K}\right)\left(\left(\frac{\Gamma_{0}}{2 \pi}\right)^{2}\right)^{\prime}\left(\frac{(\log r)(\cos 2 \theta+1)}{2}+\frac{\theta^{2}}{2}+\frac{\cos 2 \theta}{8}-\frac{\cos 4 \theta}{8}\right)\right. \\
& \left.+\frac{D_{0}^{\prime}}{4 \pi\left(K^{*}\right)^{3 / 2}}(\cos 2 \theta+1)+\frac{E_{0}^{\prime}}{4 \pi\left(K^{*}\right)^{3 / 2}} \sin 2 \theta\right\}-\frac{\Gamma_{2}\left(z^{*}\right)}{2 \pi} \theta+o\left(\frac{\log r}{r}\right),
\end{aligned}
$$

where $\Gamma_{2}\left(z^{*}\right)$, the induced circulation, is to be found, and

$$
\begin{aligned}
J_{0}\left(z^{*}\right)= & +\frac{1}{4 \pi} \int_{-\cos \beta}^{\cos \beta} \frac{\Gamma_{0}^{\prime}(s)}{z^{*}-s} d s-\frac{\tan \beta}{2 \pi \sqrt{K^{*}}}\left\{+\Gamma_{0}^{\prime}\left(1+\ln \sqrt{4 K^{*}\left(\cos ^{2} \beta-z^{* 2}\right)}\right)\right. \\
& \left.-\frac{1}{2} \int_{-\cos \beta}^{\cos \beta} \frac{\Gamma_{0}^{\prime}(s)-\Gamma_{0}^{\prime}\left(z^{*}\right)}{\left|z^{*}-s\right|} d s\right\} \\
& -\frac{\Gamma_{0}^{\prime}}{2 \pi}\left(\left(1+\frac{\tan \beta}{\sqrt{K^{*}}}\right) \ln \sqrt{K}-\ln \left(\sqrt{K^{*}}-\tan \beta\right)\right) .
\end{aligned}
$$

The conditions on the trailing edge and wake are identical to those for $\phi_{0}, \phi_{1}$, namely:

$$
\begin{gathered}
{\left[\phi_{2 \sigma}\right]_{\mathrm{TE}}=0,} \\
{\left[\phi_{2}\right]_{\mathrm{VS}}=\Gamma_{2}\left(z^{*}\right) .}
\end{gathered}
$$

The shock conditions for the forced equation are worked out in Appendix B. The results are:

$$
\begin{gathered}
{\left[K^{*} \phi_{0 \sigma}-\frac{(\gamma+1)}{2} \phi_{0 \sigma}^{2}\right]\left[\phi_{2 \sigma}\right]+\left[\phi_{0 \sigma}\right]\left[K^{*} \phi_{2 \sigma}-(\gamma+1) \phi_{0 \sigma} \phi_{2 \sigma}\right]+2\left[\phi_{0 \tilde{y}}\right]\left[\phi_{2 \tilde{y}}\right]} \\
=-g_{2}(\tilde{y})\left\{\left[K^{*} \phi_{0 \sigma \sigma}-(\gamma+1) \phi_{0 \sigma} \phi_{0 \sigma \sigma}\right]\left[\phi_{0 \sigma}\right]+\left[\phi_{0 \sigma \sigma}\right]\left[K^{*} \phi_{0 \sigma}-\frac{\gamma+1}{2} \phi_{0}^{2}\right]\right. \\
\left.+2\left[\phi_{0 \tilde{y}}\right]\left[\phi_{0 \sigma \tilde{y}}\right]+2 \tan \beta\left[\phi_{0 \sigma z^{*}}\right]\left[\phi_{0 \sigma}\right]\right\}
\end{gathered}
$$




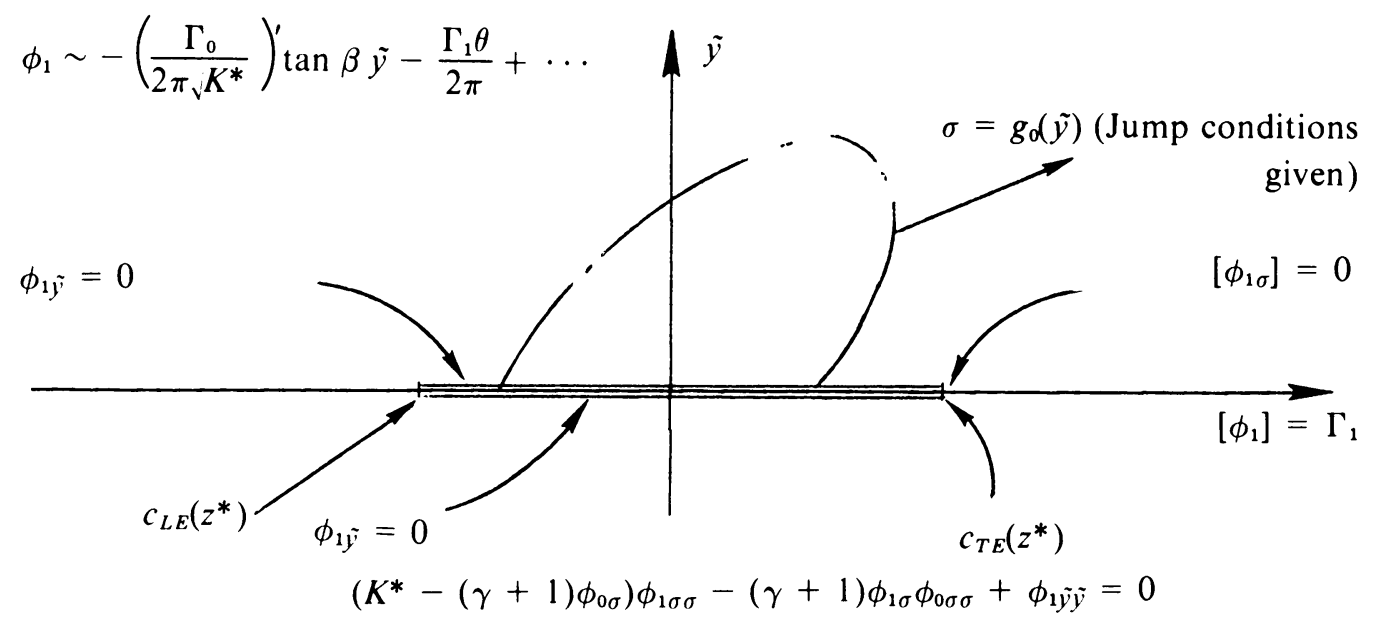

FIG. 4.2. Boundary value problem for $\phi_{1}$.

$$
\left[\phi_{2}\right]=-g_{2}\left[\phi_{0 \sigma}\right],
$$

where again all jumps are applied on the zeroth-order locus $\sigma=g_{0}(\tilde{y})$. As for $\phi_{1}$, an overall conservation theorem has been derived for $\phi_{2}$ to act as a supplementary shock condition,

$$
\int_{s_{0}}\left\{\left[w^{*} \phi_{2 \sigma}+2 \tan \beta \phi_{0 z}{ }^{*}\right]-\frac{\left[\phi_{0 \tilde{y}}\right]}{\left[\phi_{0 \sigma}\right]}\left[K^{*} \phi_{2 \tilde{y}}\right]\right\} d \tilde{y}=0 .
$$

This guarantees that there are no source terms at infinity in the solution $\phi_{2}$. Fig. 4.3 illustrates the boundary value problem for $\phi_{2}$.

Note that as in the unswept case, if the flow about the airfoil represented by $\phi_{0}$ is shock-free, then so is that represented by $\phi_{1}, \phi_{2}$. The uniqueness of the solution for the

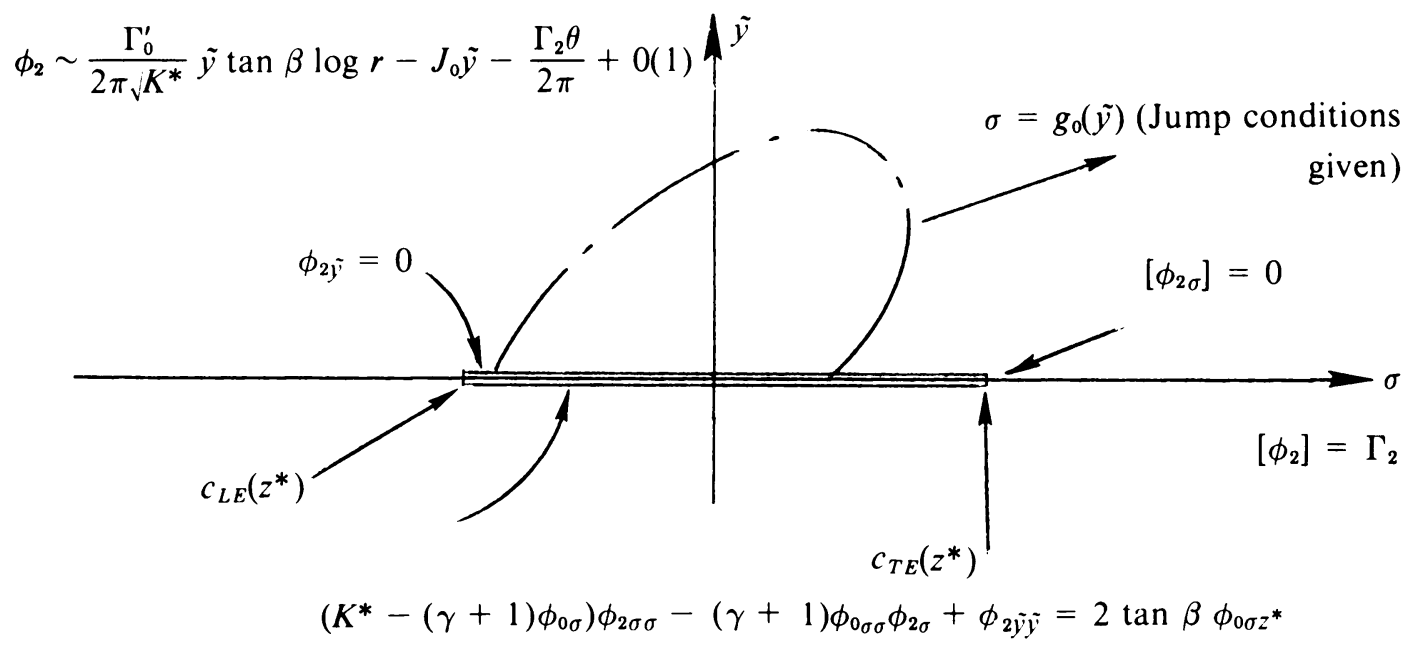


shock-free case, when $\phi_{0}$ and hence $\phi_{1}, \phi_{2}$ are shock-free, has been proved [5]. That is, if $\phi_{1,2}^{a}, \phi_{1,2}^{b}$, are two solutions of the $\phi_{l}$ problem respectively, then

$$
\Xi=\phi_{1,2}^{a}-\phi_{1,2}^{b}
$$

satisfies

$$
\begin{gathered}
\left(K^{*}-(\gamma+1) \phi_{0 \sigma}\right) \Xi_{\sigma \sigma}-(\gamma+1) \phi_{0 \sigma \sigma} \Xi_{\sigma}+\Xi_{\tilde{y} \tilde{y}}=0, \\
\left.\Xi_{\tilde{y}}\right|_{\tilde{y}=0}=0, C_{L E}\left(x^{*}\right)<\sigma<C_{T E}\left(z^{*}\right), \\
{\left[\Xi_{\sigma}\right]_{T E}=0, \Xi=-\left(\Gamma_{i} / 2 \pi\right) \theta+O(1 / r) \text { as } r \rightarrow \infty,}
\end{gathered}
$$

as in [4].

For computational ease, since the $\phi_{2}$ problem is linear, it can be broken down into two problems. We have $\phi_{2}=\phi_{2}^{(1)}+\phi_{2}^{(2)}$, where $\phi_{2}^{(1)}$ satisfies:

$$
\begin{gathered}
\left(K^{*}-(\gamma+1) \phi_{0 \sigma}\right) \phi_{2 \sigma \sigma}^{(1)}-(\gamma+1) \phi_{0 \sigma \sigma} \phi_{2 \sigma}^{(1)}+\phi_{2 y \tilde{y}}^{(1)}=0, \\
\left.\phi_{2 y}^{(1)}\right|_{\tilde{y}=0}=0, C_{L E}\left(z^{*}\right)<\sigma<C_{T E}\left(z^{*}\right), \\
{\left[\phi_{2 \sigma}^{(1)}\right]_{T E}=0,} \\
\phi_{2}^{(1)}=-\frac{\tilde{y}}{2 \pi} f \frac{\Gamma_{0}^{\prime}}{z^{*}-\xi} d \xi-\frac{\Gamma_{2}^{(1)}\left(z^{*}\right)}{2 \pi} \theta+O(1 / r) \quad \text { as } r \rightarrow \infty,
\end{gathered}
$$

and across the zeroth-order shock

$$
\begin{gathered}
{\left[K^{*} \phi_{0 \sigma}-\left(\frac{\gamma+1}{2}\right) \phi_{0 \sigma}^{2}\right]\left[\phi_{2 \sigma}^{(1)}\right]+\left[\phi_{0 \sigma}\right]\left[K^{*} \phi_{2 \sigma}^{(1)}-(\gamma+1) \phi_{0 \sigma} \phi_{2 \sigma}^{(1)}\right]+2\left[\phi_{0 \tilde{y}}\right]\left[\phi_{2 \dot{y}}^{(1)}\right]} \\
=g_{2}^{(1)}(\tilde{y})\left\{\left[K^{*} \phi_{0 \sigma \sigma}-(\gamma+1) \phi_{0 \sigma} \phi_{0 \sigma \sigma}\right]\left[\phi_{0 \sigma}\right]\right. \\
\left.+\left[K^{*} \phi_{0 \sigma}-\frac{\gamma+1}{2} \phi_{0 \sigma}^{2}\right]\left[\phi_{0 \sigma \sigma}\right]+2\left[\phi_{0 \tilde{y}}\right]\left[\phi_{0 \sigma \tilde{y}}\right]\right\}, \\
{\left[\phi_{2}^{(1)}\right]=-g_{2}^{(1)}\left[\phi_{0 \sigma}\right],}
\end{gathered}
$$

where we also have $g_{2}(\tilde{y})=g_{2}^{(1)}+g_{2}^{(2)}$, and $\phi_{2}^{(2)}$ satisfies

$$
\begin{gathered}
\left(K^{*}-(\gamma+1) \phi_{0 \sigma}\right) \phi_{2 \sigma \sigma}^{(2)}-(\gamma+1) \phi_{0 \sigma} \phi_{2 \sigma}^{(2)}+\phi_{2 \tilde{y} \tilde{y}}^{(2)}=\tan \beta \phi_{0 \sigma z^{*}}{ }^{*} \\
\left.\phi_{2 y}^{(2)}\right|_{\tilde{y}=0}=0, C_{L E}\left(z^{*}\right)<\sigma<C_{T E}\left(z^{*}\right), \\
{\left[\phi_{2 \sigma}^{(2)}\right]_{T E}=0,} \\
\phi_{2}^{(2)=} \tan \beta \frac{\Gamma_{0}}{2 \pi} \tilde{y} \log r+J_{0}^{c}\left(z^{*}\right) \tilde{y}+\tan \beta\left\{( \frac { \gamma + 1 } { 4 K } ) ( ( \frac { \Gamma _ { 0 } } { 2 \pi } ) ^ { 2 } ) ^ { \prime } \left(\frac{\log r(\cos 2 \theta+1)}{2}+\frac{\theta^{2}}{2}\right.\right. \\
\left.\left.+\frac{\cos 2 \theta}{8}-\frac{\cos 4 \theta}{8}\right)+\frac{D_{0}^{\prime}}{4 \pi\left(K^{*}\right)^{3 / 2}}(\cos 2 \theta+1)+\frac{E_{0}^{\prime}}{4 \pi\left(K^{*}\right)^{3 / 2}} \sin 2 \theta\right\} \\
-\frac{\Gamma_{2}^{(2)}\left(z^{*}\right)}{2 \pi} \theta+O\left(\frac{\log r}{r}\right) \quad \text { as } r \rightarrow \infty,
\end{gathered}
$$


where

$$
\begin{aligned}
J_{0}^{c}\left(z^{*}\right)= & -\frac{\tan \beta}{\sqrt{ } K^{*}}\left\{\frac{\Gamma_{0}^{\prime}}{2 \pi}\left(1+\frac{\ln \left\{\left(\cos ^{2} \beta-z^{* 2}\right) 4 K^{* 2}\right\}}{2}\right)\right. \\
& \left.-\frac{1}{2}\left(\int_{-\cos \beta}^{z^{*}}+\int_{\cos \beta}^{z^{*}}\right)\left(\Gamma_{0}^{\prime \prime}(s) \ln \left|z^{*}-s\right| d s\right)\right\} \\
& -\frac{\Gamma_{0}^{\prime}}{2 \pi}\left(\left(1+\frac{\tan \beta}{\sqrt{ } K^{*}}\right) \ln \sqrt{ } K-\ln \left(\sqrt{ } K^{*}-\tan \beta\right)\right) .
\end{aligned}
$$

The shock conditions for $\phi_{2}^{(2)}$ are (4.23) and (4.24), with $\phi_{2}$ replaced by $\phi_{2}^{(2)}, g_{2}$ by $g_{2}^{(2)}$.

That is, the $\phi_{2}$ problem separates into two pieces. The first piece, $\phi_{2}^{(1)}, g_{2}^{(1)}$, is precisely the correction that arises if there is no sweep. If sweep occurs, a second term $\phi_{2}^{(2)}$ must be superimposed on $\phi_{2}^{(1)}$ to correct for the sweep angle. The induced circulation then is $\left(\Gamma_{1} \log B / B\right)+\left(\Gamma_{2}^{(1)}+\Gamma_{2}^{(2)}\right)(1 / B)$.

Numerical results have been found for the unswept case by Small [10, 11]. Thus essentially $\phi_{0}, \phi_{2}^{(1)}$ are known. Computation of $\phi_{1}$ will present no new difficulties as is also probably true of $\phi_{2}^{(2)}$.

There are singularities in the small-disturbance approximations both at the tips of the airfoil and at the foot of the shock. We have not dealt with those specifically.

5. Similar sections. The planform with similar cross-sections is especially amenable to lifting line analysis since, by suitable scaling, the problems for $\phi_{2}, \phi_{1}, \phi_{2}^{(1)}$ become independent of $z^{*}$.

Consider a wing surface given by (2.1) such that the planform has a chord $c\left(z^{*}\right)$. If we assume that

$$
\frac{\partial F_{u, l}}{\partial \sigma}\left(\sigma, z^{*}\right)-A=G_{u, l}^{\prime}\left(\sigma / c\left(z^{*}\right)\right),
$$

and if we scale $\phi_{0}, \sigma, \tilde{y}$ by the chord so that

$$
\phi_{0}=c\left(z^{*}\right) \psi_{0}(\Sigma, Y), \Sigma=\frac{\sigma-\frac{1}{2}\left(C_{L E}-C_{T E}\right)}{2}, Y=\tilde{y} / c\left(z^{*}\right),
$$

then the problem (4.1), (4.2), (4.3), (4.4) for $\phi_{0}$ becomes

$$
\begin{aligned}
& \left(K^{*}-(\gamma+1) \psi_{0 \Sigma}\right) \psi_{0 \Sigma \Sigma}+\psi_{0 Y Y}=0 \text {, } \\
& \left.\psi_{0 Y}\right|_{Y=0}=G_{u, l}^{\prime}(\Sigma),-1 / 2<\Sigma<1 / 2 \text {, } \\
& {\left[\psi_{0 \Sigma}\right]_{\substack{\Sigma=1 / 2 \\
Y=0}}=0 \text {. }}
\end{aligned}
$$

This problem has no explicit dependence on $z^{*}$. The circulation $\Gamma_{0}\left(z^{*}\right)$ for $\phi_{0}$ is $\Gamma_{0}\left(z^{*}\right)=$ $c\left(z^{*}\right)\left[\psi_{0}\right]_{\substack{\Sigma=1 / 2 \\ \text {. }}}$.

A similar scaling works for $\phi_{1}, \phi_{2}^{(1)}$. For $\phi_{1}$ let $\phi_{1}=c^{\prime}\left(z^{*}\right) c\left(z^{*}\right) \psi_{0}, \Sigma, Y$ as before; the scaled $\phi_{1}$ problem from (4-8), (4-9), (4-10), (4-11), can be written

$$
\begin{gathered}
\left(\left(K^{*}-(\gamma+1) \psi_{0 \Sigma}\right) \psi_{1 \Sigma}\right)_{\Sigma}+\psi_{1 Y Y}=0, \\
\left.\psi_{1 Y}\right|_{Y=0}=0, \quad-1 / 2<\Sigma<1 / 2,
\end{gathered}
$$




$$
\begin{gathered}
{\left[\psi_{1 \Sigma}\right]_{\substack{\sum=1 / 2 \\
Y=0}}=0,} \\
\psi_{1} \sim-Y \tan \beta \frac{\left[\psi_{0}\right]}{2 \pi \sqrt{ } K^{*}}+\cdots \quad \text { as } r \rightarrow \infty .
\end{gathered}
$$

Thus, the $\phi_{1}$ problem is $z^{*}$-independent. The circulation $\Gamma_{1}\left(z^{*}\right)$ for $\phi_{1}$ is $\Gamma_{1}\left(z^{*}\right)=$ $c\left(z^{*}\right) c^{\prime}\left(z^{*}\right)\left[\psi_{1}\right]_{\substack{\Sigma=1 / 2 \\ Y=0}}$. For $\phi_{2}^{1}$, let $\phi_{2}^{(1)}=d\left(z^{*}\right) c\left(z^{*}\right) \psi_{2}^{(1)}, \psi_{0}, \Sigma, Y$ as before, where

$$
d\left(z^{*}\right)=f \frac{c^{\prime}(\xi)}{z^{*}-\xi} d \xi
$$

Then the $\psi_{2}^{(1)}$ problem can be written, from (4.26), (4.27), (4.28), (4.29), as

$$
\begin{aligned}
& \left.\left(K^{*}-(\gamma+1) \psi_{0 \Sigma}^{(1)}\right) \psi_{2 \Sigma}^{(1)}\right)_{\Sigma}+\psi_{2 Y Y}^{(1)}=0, \\
& \left.\psi_{2}^{(1)}\right|_{Y=0}=0, \quad-1 / 2<\Sigma<1 / 2 \text {, } \\
& \psi_{2}^{(1)} \sim-\frac{Y}{2}\left[\psi_{0}\right]_{\substack{\sum=1 / 2 \\
Y=0}} \text { as } r \rightarrow \infty,\left[\psi_{2}^{(1)}\right]_{\substack{\sum=1 / 2 \\
Y=0}}=0 .
\end{aligned}
$$

Thus the $\phi_{2}^{(1)}$ problem is $z^{*}$-independent.

Unfortunately the same process does not seem applicable to $\phi_{2}^{(2)}$ which is described by (4.32), (4.33), (4.34), (4.35).

Appendix A: The near field of a skewed lifting line and singular behavior of vortexline integrals. The solution of

$$
K \varphi_{0 x^{*} x^{*}}+\varphi_{0 y^{*} y^{*}}+\varphi_{0 z^{*} z^{*}}=0,
$$

on $\mathbb{R}^{3}-\left\{\left(x^{*}, z^{*}\right)\left|x^{*}-z^{*} \tan \beta>0,\right| z^{*} \mid<\cos \beta\right\}$, the equation satisfied by the first term in the outer expansion, $\varphi_{0}$, for which

$$
\varphi_{0 x^{*}} \underset{x^{*} \rightarrow \pm \infty}{\rightarrow} 0,
$$

$\left[\varphi_{0 x^{*}}\right]=0$ across $\left\{\left(x^{*}, z^{*}\right)\left|x^{*}-z^{*} \tan \beta>0,\right| z^{*} \mid<\cos \beta\right\}$, consists of a superposition of elementary horseshoe vortices distributed along $x^{*}=z^{*} \tan \beta,-\cos \beta<z^{*}<\cos \beta$, and trailing off parallel to the $x$ axis. So,

$$
\begin{aligned}
\varphi_{0}= & \frac{1}{4 \pi} \int_{-1}^{1} \frac{y^{*} \gamma(s)}{y^{* 2}+\left(z^{*}-s \cos \beta^{2}\right)^{2}} \\
& \cdot\left(1+\frac{x^{*}-s \sin \beta}{\left(\left(x^{*}-s \cos \beta\right)^{2}+K y^{* 2}+K\left(z^{*}-s \cos \beta\right)^{2}\right)^{1 / 2}}\right) d s,
\end{aligned}
$$

where $\gamma(s)$ is the distributed vorticity. At the wing tips $\gamma$ is zero, $\gamma( \pm 1)=0$.

Since we are interested in the behavior of $\varphi_{0}$ as we approach the lifting line, let $\sigma^{*}$ measure the distance from the lifting line,

$$
\sigma^{*}=x^{*}-z^{*} \tan \beta,
$$

and change variables in (A.1) so that

$$
\tau=z^{*}-s \cos \beta .
$$


Then, we are interested in the behavior, as $\sigma^{*}, y^{*} \rightarrow 0, \sigma^{*} / y^{*}$ fixed, of

$$
\begin{aligned}
& \varphi_{0}=\frac{1}{4 \pi \cos \beta}\left\{\int_{0}^{\cos \beta+z^{*}} \gamma\left(\frac{z^{*}-\tau}{\cos \beta}\right) \frac{y^{*}}{y^{* 2}+\tau^{2}} \cdot\left(1+\frac{\sigma^{*}+\tau \tan \beta}{\left(K^{*} \tau^{2}+2 \sigma^{*} \tau \tan \beta+K y^{* 2}+\sigma^{* 2}\right)^{1 / 2}}\right) d \tau\right. \\
& \left.+\int_{0}^{\cos \beta-z^{*}} \gamma\left(\frac{z^{*}-\tau}{\cos \beta}\right) \frac{y^{*}}{y^{*}+\tau^{2}} \cdot\left(1+\frac{\sigma^{*}-\tau \tan \beta}{\left(K^{*} \tau^{2}-2 \sigma^{*} \tau \tan \beta+K y^{* 2}+\sigma^{* 2}\right)^{1 / 2}}\right) d \tau\right\},
\end{aligned}
$$

where $K^{*}=K+\tan ^{2} \beta$, and the change of variables $\tau \rightarrow-\tau$ was made in the last integral. Changing variables once more in order to isolate the small parts of $\varphi_{0}$, let $\rho=\tau / y^{*}$, for $y^{*}>0$; then

$$
\varphi_{0}=\frac{1}{4 \pi \cos \beta}\left\{\int_{0}^{\infty} h_{1}\left(y^{*} \rho\right) f_{1}(\rho) d \rho+\int_{0}^{\infty} h_{2}\left(y^{*} \rho\right) f_{2}(\rho) d \rho\right\}
$$

where $h_{1}\left(y^{*} \rho\right)=\gamma\left(\left(z^{*} \mp y^{*} \rho\right) / \cos \beta\right) H\left(\cos \beta \pm z^{*}-y^{*} \rho\right), H$ is the Heaviside unit function,

$$
f_{\frac{1}{2}}(\rho)=\frac{1}{1+\rho^{2}}\left(1+\frac{A \pm C \rho}{\left(K^{*} \rho^{2} \pm D \rho+E\right)^{1 / 2}}\right),
$$

and $A=\sigma^{*} / y^{*}, C=\tan \beta, D=\left(2 \sigma^{*} / y^{*}\right) \tan \beta, E=K+\left(\sigma^{* 2} / y^{* 2}\right)$ are all fixed in the limit that $\sigma^{*}, y^{*} \rightarrow 0$.

Now (A.3) has the form for which the asymptotic expansion as $y^{*} \rightarrow 0$ can be found using Mellin transforms [1]. That is, if $M$ denotes the Mellin transform, then since

$$
\begin{aligned}
& h_{j}\left(y^{*} \rho\right) \underset{y^{*} \rho \rightarrow 0^{+}}{\sim} \gamma\left(\frac{z^{*}}{\cos \beta}\right) \mp y^{*} \rho \gamma^{\prime}\left(\frac{z^{*}}{\cos \beta}\right)+O\left(\left(y^{*} \rho\right)^{2}\right), \\
& f_{j}(\rho) \underset{\rho \rightarrow \infty}{\sim} \frac{1}{\rho^{2}}\left(1 \pm \frac{B}{\sqrt{ } E}\right)+O\left(1 / \rho^{3}\right),
\end{aligned}
$$

where ( $)^{\prime}$ means $d() / d z^{*}$, we have that

$$
M[h ; s]=\int_{0}^{\left(\cos \beta_{ \pm z^{*} / / y^{*}}\right.} \rho^{s-1} \gamma\left(\frac{z^{*} \mp y^{*} \rho}{\cos \beta}\right) d \rho
$$

is analytic for $\operatorname{Re} s>0$, and its analytic continuation to $\operatorname{Re} s>-2$ is analytic with the exception of poles at the nonpositive integers, and

$$
M\left[f_{j}: s\right]=\int_{0}^{\infty} \frac{\rho^{s-1}}{1+\rho^{2}}\left(1+\frac{A \pm C \rho}{\left(K^{*} \rho^{2} \pm D \rho+E\right)^{1 / 2}}\right) d \rho
$$

is analytic for $0<\operatorname{Re} s<2$, and its analytic continuation for $\operatorname{Re} s<3$ is analytic with the exception of a pole at $s=2$. Also,

$$
M\left[h_{j} ; 1-s\right] M\left[f_{j}: s\right] \rightarrow 0 \quad \text { as }|\operatorname{Im} s| \rightarrow \infty .
$$

Thus,

$$
\begin{aligned}
\varphi_{0} & =\frac{1}{4 \pi \cos \beta} \sum_{j=1}^{2} \int_{0}^{\infty} h_{j}\left(y^{*} \rho\right) f_{j}(\rho) d \rho \\
& =\frac{1}{4 \pi \cos \beta} \sum_{j=1}^{2} 2 \pi i \int_{\nu-i \infty}^{\nu+i \infty} M\left[h_{j} ; 1-s\right] M\left[f_{j} ; s\right] d s
\end{aligned}
$$


for $0<\nu<1$,

$$
=-\frac{1}{4 \pi \cos \beta} \sum_{j=1}^{2} \underset{s=1,2}{\operatorname{Residue}}\left(M\left[h_{j} ; 1-s\right] M\left[f_{j} ; s\right]\right)+O\left(y^{* 2} \ln y^{*}\right)
$$

as $y^{*} \rightarrow 0$. The proof can be found in Bleistein and Handelsman [1].

To find the explicit terms in the expansion note that

$$
\begin{aligned}
M\left[f_{j} ; s\right]= & \int_{0}^{\infty}\left\{\frac{\rho^{s-1}}{1+\rho^{2}}\left(1+\frac{A \pm C \rho}{\left(K^{*} \rho^{2} \pm D \rho+E\right)^{1 / 2}}\right)-\rho^{s-3}\left(1 \pm \frac{C}{\sqrt{K^{*}}}\right) H(\rho-1)\right\} d \rho \\
& +\int_{1}^{\infty} \rho^{s-3}\left(1 \pm \frac{C}{\sqrt{K^{*}}}\right) H(\rho-1) d \rho,
\end{aligned}
$$

so that

$$
\begin{aligned}
& M\left[f_{j} ; s\right]=b_{j}+O(s-1) \quad \text { as } \quad s \rightarrow 1, \\
& M\left[f_{j} ; s\right]=-\left(1 \pm \frac{C}{\sqrt{ } K^{*}}\right) \frac{1}{s-2}+d_{j}+O(s-2) \quad \text { as } \quad s \rightarrow 2,
\end{aligned}
$$

where

$$
\begin{aligned}
& b_{j}=\int_{0}^{\infty} \frac{1}{1+\rho^{2}}\left(1+\frac{A \pm C \rho}{\left(K^{*} \rho^{2} \pm D \rho+E\right)^{1 / 2}}\right) d \rho \\
& d_{j}=\int_{0}^{\infty} \frac{1}{1+\rho^{2}}\left(1+\frac{A \pm C \rho}{\left(K^{*} \rho^{2} \pm D \rho+E\right)^{1 / 2}}\right)-\frac{1}{\rho}\left(1 \pm \frac{C}{\sqrt{ } K^{*}}\right) H(\rho-1) d \rho .
\end{aligned}
$$

Similarly,

$$
\begin{aligned}
M\left[h_{j} ; 1-s\right] & =\int_{0}^{\left(\cos \beta \pm z^{*}\right) / y^{*}} \rho^{-s}\left\{\gamma\left(\frac{z^{*} \pm y^{*} \rho}{\cos \beta}\right)-\gamma\left(\frac{z^{*}}{\cos \beta}\right) \pm y^{*} \rho \gamma^{\prime}\left(\frac{z^{*}}{\cos \beta}\right)\right\} d \rho \\
& +\gamma\left(\frac{z^{*}}{\cos \beta}\right)\left(\frac{\cos \beta \pm z^{*}}{y^{*}}\right)^{1-s} \frac{1}{1-s} \mp y^{*} \gamma^{\prime}\left(\frac{z^{*}}{\cos \beta}\right)\left(\frac{\cos \beta \pm z^{*}}{y^{*}}\right)^{2-s} \frac{1}{2-s},
\end{aligned}
$$

so that

$$
\begin{aligned}
& M\left[h_{j} ; 1-s\right]=-\gamma\left(\frac{z^{*}}{\cos \beta}\right) \frac{1}{s-1}+O(1) \text { as } s \rightarrow 1, \\
& M\left[h_{j} ; 1-s\right]= \pm y^{*} \gamma^{\prime}\left(\frac{z^{*}}{\cos \beta}\right) \frac{1}{s-2}+e_{j}+O(s-2) \text { as } s \rightarrow 2,
\end{aligned}
$$

where

$$
\begin{aligned}
e_{j}= & \int_{0}^{\left(\cos \beta \pm z^{*}\right) / y^{*}} \rho^{-2}\left\{\gamma\left(\frac{z^{*} \mp y^{*} \rho}{\cos \beta}\right)-\gamma\left(\frac{z^{*}}{\cos \beta}\right) \pm y^{*} \rho \gamma^{\prime}\left(\frac{z^{*}}{\cos \beta}\right)\right\} d \rho \\
& -\gamma\left(\frac{z^{*}}{\cos \beta}\right)\left(\frac{y^{*}}{\cos \beta \pm z^{*}}\right) \mp y^{*} \gamma^{\prime}\left(\frac{z^{*}}{\cos \beta}\right) \ln \left(\frac{\cos \beta \pm z^{*}}{y^{*}}\right) .
\end{aligned}
$$


So, from (A.4), (A.5), (A.6),

$$
\begin{aligned}
\varphi_{0}= & -\frac{1}{4 \pi \cos \beta} \sum_{j=1}^{2}\left\{-b_{j} \gamma\left(\frac{z^{*}}{\cos \beta}\right) \pm d_{j} y^{*} \gamma^{\prime}\left(\frac{z^{*}}{\cos \beta}\right)-\left(1 \pm \frac{C}{\sqrt{ } K^{*}}\right) e_{j}\right\} \\
& +O\left(y^{* 2} \ln y^{*}\right) \quad \text { as } \quad y^{*} \rightarrow 0 .
\end{aligned}
$$

Integrating by parts once in $e_{j}$, using the fact that $\gamma( \pm 1)=0$, and combining terms, we get

$$
\begin{aligned}
\varphi_{0}= & -\frac{1}{4 \pi \cos \beta}\left\{-c_{0} \gamma\left(\frac{z^{*}}{\cos \beta}\right)-\frac{2 \tan \beta}{\sqrt{ } K^{*}} \gamma^{\prime}\left(\frac{z^{*}}{\cos \beta}\right) y^{*} \ln y^{*}\right. \\
& +y^{*} \int_{\left(z^{*}-\cos \beta\right) / y^{*}}^{\left(z^{*}+\cos \beta\right) / y^{*}} \frac{\gamma^{\prime}\left(\frac{z^{*}-y^{*} \rho}{\cos \beta}\right)}{\rho} d \rho \\
& +\frac{y^{*} \tan \beta}{\sqrt{K^{*}}} 2 \gamma^{\prime}\left(\frac{z^{*}}{\cos \beta}\right)+\gamma^{\prime}\left(\frac{z^{*}}{\cos \beta}\right) \ln \left(\cos ^{2} \beta-z^{* 2}\right) \\
& \left.-y^{*} \int_{-\cos \beta}^{\cos \beta} \frac{\gamma^{\prime}\left(\frac{s}{\cos \beta}\right)-\gamma^{\prime}\left(\frac{z^{*}}{\cos \beta}\right)}{\left|z^{*}-s\right|} d s+y^{*} c_{1} \gamma^{\prime}\left(\frac{z^{*}}{\cos \beta}\right)\right\}+O\left(y^{* 2} \ln y^{*}\right),
\end{aligned}
$$

where

$$
c_{0}=b_{1}+b_{2}, \quad c_{1}=d_{1}-d_{2} .
$$

These last two constants, $c_{0}, c_{1}$, can be calculated explicitly to give

$$
\begin{gathered}
c_{0}=-2 \tan ^{-1}\left(\sqrt{ } K^{*} y^{*} / \sigma^{*}\right), \\
c_{1}=\ln \left|\frac{\tan \beta-\sqrt{ } K^{*}}{\tan \beta+\sqrt{ } K^{*}}\right|-\frac{\tan \beta}{\sqrt{ } K^{*}}\left\{\ln \left(K^{*}+\frac{\sigma^{* 2}}{y^{* 2}}\right)-\ln \frac{4 K^{* 2}}{K}\right\} .
\end{gathered}
$$

So finally,

$$
\begin{aligned}
\varphi_{0}= & -\frac{\gamma\left(\frac{z^{*}}{\cos \beta}\right)}{2 \pi \cos \beta} \theta+\frac{\tan \beta}{2 \pi \sqrt{ } K^{*}} \frac{\gamma^{\prime}\left(\frac{z^{*}}{\cos \beta}\right)}{\cos \beta} y^{*} \ln r^{*}-\frac{y^{*}}{4 \pi \cos \beta} \int_{-\cos \beta}^{\cos \beta} \frac{\gamma^{\prime}\left(\frac{s}{\cos \beta}\right)}{z^{*}-s} d s \\
& -\frac{y^{*} \tan \beta}{2 \pi \sqrt{ } K^{*} \cos \beta}\left\{\gamma^{\prime}\left(\frac{z^{*}}{\cos \beta}\right)\left(1+\ln \left(\cos ^{2} \beta-z^{* 2}\right)^{1 / 2}\right)\right. \\
& \left.-\frac{1}{2} \int_{-\cos \beta}^{\cos \beta} \frac{\gamma^{\prime}\left(\frac{s}{\cos \beta}\right)-\gamma^{\prime}\left(\frac{z^{*}}{\cos \beta}\right)}{\left|z^{*}-s\right|} d s+\gamma^{\prime}\left(\frac{z^{*}}{\cos \beta}\right) \ln \frac{2 K^{*}}{\sqrt{ } K}\right\} \\
& -\frac{y^{*} \gamma^{\prime}\left(\frac{z^{*}}{\cos \beta}\right)}{2 \pi \cos \beta} \ln \left(\frac{\sqrt{ } K^{*}-\tan \beta}{\sqrt{ } K^{*}+\tan \beta}\right)^{1 / 2}+O\left(y^{* 2} \ln y^{*}\right),
\end{aligned}
$$


or more simply,

$$
\begin{aligned}
\varphi_{0}= & \frac{-\gamma\left(\frac{z^{*}}{\cos \beta}\right) \theta}{2 \pi \cos \beta}+\frac{\tan \beta}{2 \pi \sqrt{ } K^{*}} \frac{\gamma^{\prime}\left(\frac{z^{*}}{\cos \beta}\right)}{\cos \beta} y^{*} \ln r^{*} \\
& -\frac{y^{*}}{4 \pi \cos \beta} \int_{-\cos \beta}^{\cos \beta} \frac{\gamma^{\prime}\left(\frac{s}{\cos \beta}\right)}{z^{*}-s} d s-y^{*} J_{0}^{c}\left(z^{*}\right)+\cdots,
\end{aligned}
$$

where

$$
\begin{aligned}
J_{0}^{c}\left(z^{*}\right)= & \frac{\tan \beta}{2 \pi \sqrt{ } K^{*} \cos \beta}\left\{\gamma^{\prime}\left(\frac{z^{*}}{\cos \beta}\right)\left(1+\ln \left(4 K^{* 2}\left(\cos ^{2} \beta-z^{* 2}\right)\right)^{1 / 2}\right)\right. \\
& \left.+\frac{1}{2}\left(\int_{0}^{\cos \beta}+\int_{0}^{-\cos \beta}\right)\left(\frac{\gamma^{\prime}\left(\frac{s}{\cos \beta}\right)-\gamma^{\prime}\left(\frac{z^{*}}{\cos \beta}\right)}{z^{*}-s}\right)\right\} d s \\
& +\frac{\gamma^{\prime}\left(\frac{z^{*}}{\cos \beta}\right)}{2 \pi \cos \beta}\left\{-\ln \left(\sqrt{ } K^{*}-\tan \beta\right)+\left(1+\frac{\tan \beta}{\sqrt{ } K^{*}}\right) \ln \sqrt{ } K\right\}
\end{aligned}
$$

The other integrals in (3.9), (3.10) have the form $\sigma^{*} / 4 \pi$, or $y^{*} / 4 \pi$ times the form

$$
\psi=\int_{-1}^{1} \frac{g(s)}{\left(\sigma^{* 2}+K y^{* 2}+K^{*}\left(z^{*}-s \cos \beta\right)^{2}+2 \sigma^{*}\left(z^{*}-s \cos \beta\right) \tan \beta\right)^{3 / 2}} d s .
$$

This integral can be treated in precisely the same way as $\varphi_{0}$. With $z^{*}-s \cos \beta=\tau$,

$$
\psi=\frac{1}{\cos \beta}\left(\int_{z^{*}-\cos \beta}^{z^{*+}+\cos \beta} \frac{g\left(\frac{z^{*}-\tau}{\cos \beta}\right)}{\left(\sigma^{* 2}+K y^{* 2}+K \tau^{2}+2 \sigma^{*} \tau \tan \beta\right)^{3 / 2}} d \tau\right) .
$$

With $\rho=\tau / y^{*} ; D, E$ as before,

$$
\begin{aligned}
\psi & =\frac{y^{*-2}}{\cos \beta}\left(\int_{0}^{\left(z^{*}+\cos \beta\right) / y^{*}} \frac{g\left(\frac{z^{*}-y^{*} \rho}{\cos \beta}\right)}{\left(K^{*} \rho^{2}+D \rho+E\right)^{3 / 2}} d \rho+\int_{0}^{\left(\cos \beta-z^{*}\right) / y^{*}} \frac{g\left(\frac{z^{*}+y^{*} \rho}{\cos \beta}\right)}{\left(K^{*} \rho^{2}-D \rho+E\right)^{3 / 2}} d \rho\right) \\
& =\frac{y^{*-2}}{\cos \beta}\left(\sum_{j=1}^{2} \int_{0}^{\infty} \mathcal{F}_{j}\left(y^{*} \rho\right) F_{j}(\rho) d \rho\right),
\end{aligned}
$$

where

$$
\underset{2}{\mathfrak{F}_{1}\left(y^{*} \rho\right)}=g\left(\frac{z^{*} \mp y^{*} \rho}{\cos \beta}\right) H\left(\cos \beta \pm z^{*}-\rho\right), \quad \mathcal{F}_{1}(\rho)=\frac{1}{\left(K^{*} \rho^{2} \pm D \rho+E\right)^{3 / 2}} .
$$


Then

so that

$$
\mathcal{H}_{j}\left(y^{*} \rho\right) \underset{y^{*} \rho \rightarrow 0^{+}}{\sim} g\left(\frac{z^{*}}{\cos \beta}\right)+O\left(y^{*} \rho\right), \quad \mathcal{F}_{j}(\rho) \underset{\rho \rightarrow \infty}{\sim} \frac{1}{\left({ }^{*} K^{*} \rho\right)^{3}}+O\left(1 / \rho^{4}\right),
$$

$$
M\left[\mathcal{H}_{j} ; s\right]=\int_{0}^{\cos \beta \pm z^{*} / y^{*}} \rho^{s-1} g\left(\frac{z^{*} \mp y^{*} \rho}{\cos \beta}\right) d \rho
$$

is analytic for $\operatorname{Re} s>0$ and its analytic continuation to the negative half plane is analytic except for poles at the negative integers,

$$
M\left[\mathcal{F}_{j} ; s\right]=\int_{0}^{\infty} \frac{\rho^{s-1}}{\left(K^{*} \rho^{2} \pm D \rho+E\right)^{3 / 2}} d \rho
$$

is analytic for $-0<\operatorname{Re} s<3$ and its analytic continuation to the whole plane has poles at the integers, and

$$
M\left[\mathcal{H}_{j} ; 1-s\right] M\left[\mathfrak{F}_{j} ; s\right] \underset{\operatorname{Im} s \rightarrow \infty}{\longrightarrow} 0
$$

So,

$$
\begin{aligned}
\psi & =\frac{y^{*-2}}{\cos \beta}\left(\sum_{j=1}^{2} \int_{0}^{\infty} \mathfrak{K}_{j}\left(y^{*} \rho\right) \mathcal{F}_{j}(\rho) d \rho\right) \\
& =\frac{y^{*-2}}{\cos \beta}\left(\sum_{j=1}^{2} 2 \pi i \int_{\nu-i \infty}^{\nu+i \infty} M\left[\mathfrak{H}_{;} ; 1-s\right] M\left[\mathfrak{F}_{j} ; s\right] d s\right)
\end{aligned}
$$

for $0<\nu<1$,

$$
=\frac{-y^{*-2}}{\cos \beta} \sum_{j=1}^{2} \underset{s=1,2}{\text { Residue }} M\left[\mathfrak{H}_{j} ; 1-s\right] M\left[\mathfrak{F}_{j} ; s\right]+O\left(y^{*} \ln y^{*}\right)
$$

as $y^{*} \rightarrow 0$.

Since

$$
M\left[\mathcal{K}_{j}, 1-s\right]=\int_{0}^{\cos \beta \pm z^{*}, l^{*}} \rho^{-s} g\left(\frac{z^{*} \mp y^{*} \rho}{\cos \beta}\right) d \rho
$$

then

$$
M\left[\mathcal{H C}_{j} ; 1-s\right]=\frac{-g\left(z^{*} / \cos \beta\right)}{s-1}+O(1) \quad \text { as } \quad s \rightarrow 1
$$

and

$$
M\left[\mathfrak{K}_{j} ; 1-s\right]= \pm \frac{y^{*} g^{\prime}\left(z^{*} \cos \beta\right)}{s-2}+O(1) \text { as } \quad s \rightarrow 2 .
$$

So, from (A.10), (A.11), (A.12),

$$
\psi=\frac{2 \sqrt{K^{*}}}{K \cos \beta} \frac{1}{r^{* 2}} g\left(z^{*} / \cos \beta\right)+\frac{1}{K_{\sqrt{ }} K^{*}} \tan \beta \frac{\sigma}{r^{* 2}} g^{\prime}\left(z^{*} / \cos \beta\right)+O\left(\ln y^{*}\right) .
$$

So, (3.9) is

$$
\phi_{1}=\frac{\sigma^{*}}{2 \pi r^{* 2} K^{*}} \frac{\mathscr{D}_{1}\left(z^{*} / \cos \beta\right)}{\cos \beta}+\frac{\sigma^{* 2}}{2 \pi r^{* 2}} \frac{\tan \beta}{K^{* 3 / 2}} \frac{\mathscr{D}_{1}^{\prime}}{\cos \beta}+O\left(y^{*} \ln y^{*}\right)
$$


as $y^{*} \rightarrow 0$, and $(3.10)$ is

$$
\begin{aligned}
\phi_{2} & =\frac{\sigma^{*}}{2 \pi \sqrt{ } K^{*} r^{* 2}} \frac{D_{2}\left(z^{*} / \cos \beta\right)}{\cos \beta}+\frac{y^{*}}{2 \pi \sqrt{ } K^{*} r^{* 2}} \frac{\mathcal{E}_{2}\left(z^{*} / \cos \beta\right)}{\cos \beta} \\
& -\frac{\gamma_{2} \theta}{2 \pi \cos \beta}+\phi_{2}^{P}+\frac{\sigma^{*} \tan \beta}{2 \pi r^{* 2}\left(K^{*}\right)^{3 / 2}} \frac{D_{2}^{\prime}}{\cos \beta}+\frac{y^{* 2} \tan \beta}{2 \pi r^{* 2}\left(K^{*}\right)^{3 / 2}} \frac{\mathcal{E}_{2}^{\prime}}{\cos \beta} \\
& +O\left(y^{*} \ln y^{*}\right)
\end{aligned}
$$

as $y^{*} \rightarrow 0$.

Appendix B: Shock relations. The shock conditions for $\phi(x, \tilde{y}, \tilde{z} ; B)$ are the conservation form of the full equation (2.3),

$$
\left[K \phi_{x}-\frac{\gamma+1}{2} \phi_{x}^{2}\right]_{s}\left[\phi_{x}\right]_{s}+\left[\phi_{\bar{y}}\right]_{s}^{2}+\left[\phi_{z}\right]_{s}^{2}=0,
$$

and the condition of no tangential jump across the shock,

$$
[\phi]_{8}=0 \text {, }
$$

where the shock locus is given by

$$
x=G(\tilde{y}, \tilde{z} ; B) .
$$

In the new coordinates $\sigma=x-\tilde{z} \tan \beta, \tilde{y}, \tilde{z}$, these conditions become

$$
\begin{gathered}
{\left[K^{*} \phi_{\sigma}-\frac{\gamma+1}{2} \phi_{\sigma}^{2}\right]_{s}\left[\phi_{\sigma}\right]_{s}+\left[\phi_{\bar{y}}\right]_{s}^{2}+\left[\phi_{\bar{z}}\right]_{s}^{2}-2 \tan \beta\left[\phi_{\bar{z}}\right]_{s}\left[\phi_{\sigma}\right]_{s}=0,} \\
{[\phi]_{s}=0,}
\end{gathered}
$$

where the shock locus is given by

$$
\sigma=g(\tilde{y}, \tilde{z} ; B)=-\tilde{z} \tan \beta+G(\tilde{y}, \tilde{z} ; B) .
$$

In inner variables $\sigma, \tilde{y}, z^{*}=\tilde{z} / B$, the expansions of $\phi$, and of the shock location $S$, are

$$
\begin{gathered}
\phi=\phi_{0}\left(\sigma, \tilde{y}, z^{*}\right)+\frac{\log B}{B} \phi_{1}\left(\sigma, \tilde{y}, z^{*}\right)+\frac{1}{B} \phi_{2}\left(\sigma, \tilde{y}, z^{*}\right)+\cdots, \\
\sigma=g_{0}\left(\tilde{y}, z^{*}\right)+\frac{\log B}{B} g_{1}\left(\tilde{y}, z^{*}\right)+\frac{1}{B} g_{2}\left(\tilde{y}, z^{*}\right)+\cdots .
\end{gathered}
$$

For any function $f(x, \tilde{y}, \tilde{z} ; B)$ with an expansion of the form (B.6),

$$
\begin{aligned}
{[f]_{s}=f\left(\left(g_{0}+\frac{\log B}{B} g_{1}+\frac{1}{B} g_{2}+\cdots\right)^{+}, \tilde{y}, \tilde{z} ; B\right) } & \\
& -f\left(\left(g_{0}+\frac{\log B}{B} g_{1}+\frac{1}{B} g_{2}+\cdots\right)^{-}, \tilde{y}, \tilde{z} ; B\right) .
\end{aligned}
$$

So, expanding $f$ in terms of the $f_{t}$ for large $B$, and expanding the $f_{t}$ in their right- and lefthand Taylor series about $g_{0}$, we obtain

$$
[f]_{s}=\left[f_{0}\right]_{s_{0}}+\frac{\log B}{B}\left\{g_{1}\left[f_{0 \sigma}\right]_{s_{0}}+\left[f_{1}\right]_{s_{0}}\right\}+\frac{1}{B}\left\{g_{2}\left[f_{0 \sigma}\right]_{s_{0}}+\left[f_{2}\right]_{s_{0}}\right\}+\cdots .
$$


Substituting the appropriate expansions of the form (B.6), (B.7) into the shock relations (B.3), (B.4) gives

$$
\begin{aligned}
& \left\{\left[K^{*} \phi_{0 \sigma}-\frac{\gamma+1}{2} \phi_{0 \sigma}^{2}\right]_{s_{0}}+\frac{\log B}{B}\left[K^{*} \phi_{1 \sigma}-(\gamma+1) \phi_{0 \sigma} \phi_{1 \sigma}\right]_{s_{0}}\right. \\
& \left.+\frac{1}{B}\left[K^{*} \phi_{2 \sigma}-(\gamma+1) \phi_{0 \sigma} \phi_{2 \sigma}\right]_{s_{0}}+\left(\frac{\log B}{B} g_{1}+\frac{1}{B} g_{2}\right)\left[K^{*} \phi_{0 \sigma \sigma}-(\gamma+1) \phi_{0 \sigma} \phi_{0 \sigma \sigma}\right]_{s_{0}}\right\} \\
& \cdot\left\{\left[\phi_{0 \sigma}\right]_{s_{0}}+\frac{\log B}{B}\left[\phi_{1 \sigma}\right]_{s_{0}}+\frac{1}{B}\left[\phi_{2 \sigma}\right]_{s_{0}}+\left(\frac{\log B}{B} g_{1}+\frac{1}{B} g_{2}\right)\left[\phi_{0 \sigma \sigma}\right]_{s_{0}}\right\} \\
& +\left[\phi_{0 \tilde{y}}\right]_{s_{0}}^{2}+\frac{2 \log B}{B}\left[\phi_{0 \tilde{y}}\right]_{s_{0}}\left[\phi_{1 \tilde{y}}\right]_{s_{0}}+2 \frac{1}{B}\left[\phi_{2 \tilde{y}}\right]_{s_{0}}\left[\phi_{0 \tilde{y}}\right]_{s_{0}} \\
& +\left(\frac{\log B}{B} g_{1}+\frac{1}{B} g_{2}\right) 2\left[\phi_{0 y}\right]_{s_{0}}\left[\phi_{0 \sigma y}\right]_{s_{0}}-\frac{2 \tan \beta}{B}\left[\phi_{0 z^{*}}\right]_{s_{0}}\left[\phi_{0 \sigma}\right]_{s_{0}}+O\left(\left(\frac{\log B}{B}\right)^{2}\right)=0,
\end{aligned}
$$

and

$$
\left[\phi_{0}\right]_{s_{0}}+\frac{\log B}{B}\left[\phi_{1}\right]_{s_{0}}+\frac{1}{B}\left[\phi_{2}\right]_{s_{0}}+\left(\frac{\log B}{B} g_{1}+\frac{1}{B} g_{2}\right)\left[\phi_{0 \sigma}\right]_{s_{0}}+O\left(\left(\frac{\log B}{B}\right)^{2}\right)=0 \text {. }
$$

Collecting terms of the same order gives:

$$
\begin{gathered}
O(1):\left[K^{*} \phi_{0 \sigma}-\frac{\gamma+1}{2} \phi_{0 \sigma}^{2}\right]_{s_{0}}\left[\phi_{0 \sigma}\right]_{s_{0}} \cdot\left[\phi_{0 y} \tilde{y}\right]_{s_{0}}^{2}=0, \\
{\left[\phi_{0}\right]_{s_{0}}=0,}
\end{gathered}
$$

$$
\begin{gathered}
O\left(\frac{\log B}{B}\right):\left[K^{*} \phi_{0 \sigma}-\frac{\gamma+1}{2} \phi_{0 \sigma}^{2}\right]_{s_{0}}\left[\phi_{1 \sigma}\right]_{s_{0}}+\left[K^{*} \phi_{1 \sigma}-(\gamma+1) \phi_{0 \sigma} \phi_{1 \sigma}\right]_{s_{0}}\left[\phi_{0 \sigma}\right]_{s_{0}} \\
+g_{1}\left\{\left[K^{*} \phi_{0 \sigma \sigma}-(\gamma+1) \phi_{0_{0}} \phi_{0 \sigma \sigma 0}\right]\left[\phi \phi_{0 \sigma} \phi_{0 \sigma}\right]_{s_{0}}+\left[\phi_{0 \sigma \sigma}\right]_{s_{0}}\left[K^{*} \phi_{0 \sigma}-\frac{\gamma+1}{2} \phi_{0 \sigma}^{2}\right]_{s_{0}}\right. \\
\left.\left.+2\left[\phi_{0 \tilde{y}}\right]\right]_{s_{0}}\left[\phi_{1 \tilde{y}}\right]\right]_{s_{0}}+2 g_{1}\left[\phi_{0 y}\right]_{s_{0}}\left[\phi_{0 \sigma \tilde{y}}\right]_{s_{0}}=0 \\
{\left[\phi_{1}\right]_{s_{0}}=-g_{1}\left[\phi_{0 \sigma}\right]_{s_{0}},}
\end{gathered}
$$

$$
\begin{gathered}
O\left(\frac{1}{B}\right):\left[K^{*} \phi_{0 \sigma}-\frac{\gamma+1}{2} \phi_{0 \sigma}^{2}\right]_{s_{0}}\left[\phi_{2 \sigma}\right]_{s_{0}}+\left[K^{*} \phi_{2 \sigma}-(\gamma+1) \phi_{2 \sigma}\right]_{s_{0}}\left[\phi_{0 \sigma}\right]_{s_{0}}+2\left[\phi_{0 \dot{y}}\right]_{s_{0}}\left[\phi_{2 \dot{y}}\right]_{s_{0}} \\
=g_{2}\left\{\left[K^{*} \phi_{0 \sigma \sigma}-(\gamma+1) \phi_{0 \sigma} \phi_{0 \sigma \sigma}\right]_{s_{0}}\left[\phi_{0 \sigma}\right]_{s_{0}}+\left[\phi_{0 \sigma \sigma}\right]_{s_{0}}\right. \\
\\
{\left[K^{*} \phi_{0 \sigma}-\frac{\gamma+1}{2} \phi_{0 \sigma}^{2}\right]_{s_{0}}+2\left[\phi_{0 \tilde{\sigma} \tilde{y}] s_{0}}\left[\phi_{0 \tilde{y}}\right]_{s_{0}}+2 \tan \beta\left[\phi_{0 z^{*}}\right]_{s_{0}}\left[\phi_{0 \sigma}\right]_{s_{0}},\right.} \\
{\left[\phi_{2}\right]_{s_{0}}=-g_{2}\left[\phi_{0 \sigma}\right]_{s_{0}}}
\end{gathered}
$$

The zeroth-order conditions (B.8), (B.9) are the usual two-dimensional shock conditions where $K^{*}$ is the adjusted similarity parameter. The $O(\log B / B)$ conditions (B.10), (B.11) 
involve three unknowns, $\left[\phi_{1 \sigma}\right]_{s_{0}},\left[\phi_{1 y}\right]_{\delta_{0}}, g_{1}$, and are essentially the same as the first-order correction for the unswept case (which is $O(1 / B)$ ). The $O(1 / B)$ correction is the same as the $O(\log B / B)$ correction except that the extra term $-2 \tan \beta\left[\phi_{02^{*}}\right]_{0_{0}}\left[\phi_{0 \sigma}\right]_{s_{0}}$ turns up to correct for the sweep angle.

As in the unswept case, there is another shock condition to be checked which arises by applying Green's Theorem to the equations for $\phi_{1}, \phi_{2}$, which are in divergence forms. The equation, shock conditions, and boundary conditions for $\phi_{1}$ are the same as those for the first-order correction in the nonswept case, hence [5]:

$$
\begin{aligned}
0 & =\iint_{D} \dot{\nabla} \cdot\left(-w^{*} \phi_{1 \sigma}, K^{*} \phi_{1}\right) d \sigma d \hat{y}=\oint_{D}\left(-w^{*} \phi_{1 \sigma}, K^{*} \phi_{1} \tilde{y}\right) \cdot \hat{n} d l \\
& =\int_{s_{0}} w^{*} \phi_{1 \sigma} d \hat{y}+K^{*} \phi_{1 \tilde{y}} d \sigma, \quad \hat{y}=\sqrt{K} \tilde{y}, \hat{\nabla}=\left(\frac{\partial}{\partial \sigma}, \frac{\partial}{\partial \tilde{y}}\right),
\end{aligned}
$$

where $D$ is the region bounded by the body $B$, the zeroth-order shock $S_{0}$, the wake $W$. The integrals over $B, W$ and $S_{0}$ vanish from the boundary conditions. Since $d \tilde{y} / d \sigma=-\left[\phi_{0 \sigma}\right]_{s_{0}} /$ $\left[\phi_{0 y}\right]_{s_{0}}$, we have

$$
\int_{S_{0}}\left\{\left[w^{*} \phi_{1 \sigma}\right]-\frac{\left[\phi_{0 \tilde{y}}\right]}{\left[\phi_{0 \sigma}\right]}\left[\phi_{1 \tilde{y}}\right]\right\} d \tilde{y}=0
$$

For $\phi_{2}$,

$$
\begin{aligned}
0= & \iint_{D} \hat{\nabla} \cdot\left(\left(w^{*} \phi_{2 \sigma}-2(\tan \beta) \phi_{0 z^{*}}\right), K^{*} \phi_{2 \hat{y}}\right) d \sigma d \hat{y} \\
= & \int_{B} K^{*} \phi_{2 \hat{y}} d \sigma+\int_{W} K^{*} \phi_{2 \hat{y}} d \sigma+\int_{S_{0}}\left(-w^{*} \phi_{2 \sigma}-2 \tan \beta \phi_{0 z^{*}}, K^{*} \phi_{2 \hat{y}}\right) \cdot \hat{n} d l \\
& +\int_{S_{R}}\left(\left(-w^{*} \phi_{2 \sigma}-2(\tan \beta) \phi_{0 z^{*}}\right) \sigma+K^{*} \phi_{2 \hat{y} \hat{y}}\right) d \theta
\end{aligned}
$$

where $S_{R}=\sigma^{2}+\hat{y}^{2}=R$. The integral over the body is zero since $\left.\phi_{2 \hat{y}}\right|_{B}=0$; the integral over the wake is zero since $\left[\phi_{2} \hat{y}\right]_{w}=0$. Since

$$
\begin{aligned}
& \phi_{2} \sim A \hat{y} \ln \hat{r}+J \hat{y}+B \theta+\cdots \\
& -w^{*} \phi_{2 \sigma} \sigma+K^{*} \phi_{1} \hat{y} \hat{y}-2 \tan \beta \phi_{0 z^{*}} \sigma \\
& \sim+\left(K^{*}+(\gamma+1) \Gamma \frac{\hat{y}}{r^{2}}\right)\left(\frac{A \hat{y} \sigma^{2}}{r^{2}}-\frac{B \hat{y} \sigma}{\hat{r}^{2}}\right)+K^{*} A \hat{y} \ln \hat{r}+\frac{K^{*} A \hat{y}^{3}}{\hat{r}^{2}} \\
& +J K^{*} \hat{y}+\frac{B x}{\hat{r}^{2}} K^{*} \hat{y}-2(\tan \beta) \sigma \Gamma_{0}^{\prime}+\cdots \\
& \sim K^{*} A \hat{y}+K^{*} A \hat{y} \ln \hat{r}+\frac{(\gamma+1) \Gamma A \hat{y}^{2} \sigma^{2}}{\hat{r}^{*}}-\frac{B \hat{y} \sigma K^{*}}{\hat{r}^{2}}-\frac{(\gamma+1) \Gamma B \hat{y}^{2} \sigma}{\hat{r}^{*}} \\
& +J K^{*} \hat{y}+\frac{B x \hat{y} K^{*}}{\hat{r}^{2}}-2(\tan \beta) \sigma \Gamma_{0}{ }^{\prime} \theta+\cdots, \\
& \cdot \int_{S_{R}}\left(\left(-w^{*} \phi_{2 \sigma}-2 \tan \beta \phi_{0 z^{*}}\right) \sigma+K^{*} \phi_{2 y} \hat{y}\right) d \theta \\
& =O\left(\frac{1}{R}\right)-2 \tan \beta \Gamma_{0}{ }^{\prime}\left(z^{*}\right) \int_{0}^{2 \pi} R \theta \cos \theta d \theta_{R} \simeq_{\infty} 0 .
\end{aligned}
$$


So we have

$$
\int_{S_{0}}\left(-w^{*} \phi_{2 \sigma}-2 \tan \beta \phi_{0 z^{*}}, K^{*} \phi_{2 \hat{y}}\right) \cdot \hat{n} d l=0
$$

or

$$
\int_{S_{0}}\left[w^{*} \phi_{2 \sigma}+2 \tan \beta \phi_{0 z^{*}}\right]-\frac{\left[\phi_{0 \hat{y}}\right]}{\left[\phi_{0 \sigma}\right]}\left[K^{*} \phi_{2 \hat{y}}\right] d \hat{y}=0 .
$$

\section{REFERENCES}

[1] N. Bleistein and R. Handelsman, Asymptotic expansions of integrals, Holt, Rinehart and Winston, New York, 1975

[2] J. W. Boerstoel and G. H. Huizing, Transonic shock-free airfoil design by an analytic hodograph method, AIAA paper No. 74-539, presented AIAA 7th Fluid and Plasma Dynamic Conf. 1974

[3] H. K. Cheng and M. M. Hafez, Transonic equivalence rule: a nonlinear problem involving lift, J. Fluid Mech. 72, 161-187 (1975)

[4] Julian D. Cole, Modern developments in transonic flow, S.I.A.M. J. Appl. Math. 29, 763-787 (1975)

[5] L. Pamela Cook, $A$ uniqueness proof for a transonic flow problem, Indiana Univ. Math. J. 27, 51-71 (1978)

[6] L. Pamela Cook and Julian D. Cole, Lifting line theory for transonic flow, S.I.A.M. J. Appl. Math. 27, 1978

[7] William Fredrick Durand, ed., Aerodynamic theory: a general review of progress; Vol. II, Division E, General aerodynamic theory, perfect fluids, Th. von Karman and J. M. Burgers, 1943, Durand Reprinting Committee, Calif. Inst. of Technology, pp. 197-201

[8] P. Garabedian and D. G. Korn, Analysis of transonic airfoils, Comm. Pure Appl. Math. 24, 848-851 (1971)

[9] E. M. Murman and J. D. Cole, Inviscid drag at transonic speeds: studies in transonic flow III, UCLA School of Eng. Rpt. 7603, Dec. 1974; also AIAA paper No. 75-540, presented at AIAA 7th Fluid and Plasma Dynamic Conference, June 1974

[10] R. D. Small, Transonic lifting line theory: numerical procedure for shock-free flows, to appear, AIAA J., June 1978

[11] R. D. Small, Calculation of a transonic lifting line theory, Studies in Transonic Flow VI, UCLA School of Eng. and Applied Science Report, April 1978

[12] James K. Thurber, An asymptotic method for determining the lift distribution of a swept-back wing of finite span. Comm. Pure Applied Math. XVIII, 733-756 (1965)

[13] M. D. Van Dyke, Perturbation methods in fluid mechanics, Parabolic Press, 1975 